\title{
Role of inductive electric fields and currents in dynamical ionospheric situations
}

\author{
H. Vanhamäki, O. Amm, and A. Viljanen \\ Finnish Meteorological Institute, Space Research Unit, P.O. Box 503, 00101 Helsinki, Finland \\ Received: 9 October 2006 - Revised: 16 January 2007 - Accepted: 8 February 2007 - Published: 8 March 2007
}

\begin{abstract}
We study the role of ionospheric induction in different commonly observed ionospheric situations. These include an intensifying electrojet, westward travelling surge (WTS) and $\Omega$-band. We use data based, realistic models for these phenomena and calculate the inductive electric fields that are created due to the temporal variations of ionospheric currents. The ionospheric induction problem is solved using a new calculation technique that can handle non-uniform, time-dependent conductances and electric fields of any geometry. We find that in some situations inductive effects are not negligible and the ionospheric electric field is not a pure potential field, but has a significant induced rotational part. In the WTS and $\Omega$-band models the induced electric field is concentrated in a small area, where the time derivatives are largest. In the electrojet model the induced field is significant over a large part of the jet area. In these examples the induced electric field has typical values of few $\mathrm{mV} / \mathrm{m}$, which amounts to several tens of percents of the potential electric field present at the same locations. The induced electric field is associated with ionospheric and field aligned currents (FAC), that modify the overall structure of the current systems. Especially the induced FAC are often comparable to the non-inductive FAC, and may thus modify the coupling between the ionosphere and magnetosphere in the most dynamical situations. We also present some examples with very simple ionospheric current systems, where the effect of different ionospheric parameters on the induction process is studied.
\end{abstract}

Keywords. Ionosphere (Electric fields and currents) - Electromagnetics (Electromagnetic theory)

Correspondence to: H. Vanhamäki

(heikki.vanhamaki@fmi.fi)

\section{Introduction}

In this paper, we study the role of inductive electric fields and currents in several common ionospheric phenomena, including an intensifying electrojet, westward travelling surge (WTS) and $\Omega$-band. Usually it is assumed that the ionospheric electric field is a potential field, so that $\nabla \times \boldsymbol{E}=0$, and it is well established that this is indeed the case in most situations (e.g. Untiedt and Baumjohann, 1993). However, Yoshikawa and Itonaga (1996) were the first to study how the inductive processes influence the reflection of Alfvén waves from the ionosphere. They found that when an incident shear Alfvén wave carrying a potential electric field is reflected from the non-isotropically conducting ionosphere, the reflected wave consists of both shear and fast mode waves. The fast mode wave is directly related to the induced rotational part of the ionospheric electric field, and also the reflected shear mode wave is modified when inductive phenomena are included in the analysis. Later studies by e.g. Buchert and Budnik (1997), Buchert (1998), Yoshikawa and Itonaga (2000), Lysak and Song (2001), Lysak (2004) and Sciffer et al. (2004), have confirmed these results and investigated further the reflection process and the propagation of the shear and fast mode waves in the ionosphere.

Previous studies have formulated the ionospheric induction problem in terms of Alfvén wave reflections and either derived analytical reflection coefficients at the ionospheric boundary (e.g. Yoshikawa and Itonaga, 1996; Buchert, 1998; and Sciffer et al., 2004), or solved numerically the propagation of Alfvén waves in and above the ionosphere (e.g. Lysak, 2004). This approach has resulted in a better understanding of the ionospheric inductive phenomena and coupling of the magnetosphere and ionosphere by Alfvén waves. However, in all these studies it is assumed that the spatial and temporal structure of the incident Alfvén waves is known. This is quite a restrictive assumption in practical studies, because the incident wave pattern is very hard to measure. To the authors'

Published by Copernicus GmbH on behalf of the European Geosciences Union. 
knowledge there seems to be no empirical models of Alfvén wave patterns related to some specific ionospheric events. In principle one could use a magnetospheric MHD simulation as an input in the Alfvén wave scheme, but in practise magnetospheric simulations use electrostatic ionospheric solvers and it would not be straightforward to couple them to an ionospheric Alfvén wave solver (Janhunen, 1998). In addition, also magnetospheric MHD simulations have problems in producing specific ionospheric phenomena.

Recently, Vanhamäki et al. (2005) used a different approach that allowed them to use purely ionospheric quantities as input, instead of incident waves. They showed by approximate calculations that inductive electric fields associated with some very dynamic ionospheric phenomena, including WTS, $\Omega$-band and Giant Pulsation, are locally very significant. These local "hot-spots" tended to occur in those areas where the field aligned currents (FAC) were largest, so in these areas the inductive processes could well contribute to the ionosphere-magnetosphere coupling. Vanhamäki et al. (2005) calculated the inductive fields caused by selfinduction in the ionosphere (primary process) and also by the ground induced currents flowing in the conducting ground (secondary process). They concluded that at ionospheric altitudes the secondary contribution from ground induction is always very small and smoothly distributed and in practice negligible when compared to the larger and more concentrated primary contibution from ionospheric self-induction.

However, the calculation method used by Vanhamäki et al. (2005) was rather approximate, giving only order of magnitude estimates. The induced electric fields in the ionosphere were calculated as vacuum fields, i.e. the currents driven by the induced fields themselves were neglected. This approximation probably gives too large induced electric fields, as the effect of the neglected current should tend to decrease the induced fields according to Lenz's law. Vanhamäki et al. (2006) presented a new calculation method that solves the ionospheric induction problem self-consistently using only ionospheric potential electric field and conductances as input. This calculation method can handle non-uniform, timedependent ionospheric conductances and electric fields of any geometry. In this paper we apply the new calculation method to several commonly observed ionospheric phenomena that have strong temporal variations. Our examples include the previously studied WTS and $\Omega$-bands systems and also an intensifying electrojet. In Sects. 2-3 we briefly outline the calculation method and discuss the general properties of ionospheric induction. In Sect. 4 we present the main results for the realistic, data-based models. Section 5 is summary and conclusions.

\section{Theory}

The calculation method has been presented by Vanhamäki et al. (2006), and here we give just a brief summary. We use a cartesian coordinate system where the ionospheric current sheet is taken to be the xy-plane and the z-axis points vertically downwards. The Earth's magnetic field is assumed to be parallel to the z-axis, which is a reasonable approximation in the northern auroral region. We also use the thinsheet approximation, i.e. we assume that all horizontal currents flow at a thin sheet at altitude $z=0$. We concentrate on the effects of ionospheric self-induction, so we do not include the induction effects that take place in the conducting Earth. While the ground induction has large effects on the electric and magnetic fields at the Earth's surface, Vanhamäki et al. (2005) concluded that at ionospheric altitudes the ground effect should be negligible when compared to the effects of ionospheric self-induction.

The input quantities in the calculation method presented by Vanhamäki et al. (2006) are the 2-dimensional distributions of ionospheric Pedersen and Hall conductances, $\Sigma_{P}$ and $\Sigma_{H}$, respectively, and the potential part of the ionospheric electric field, $\boldsymbol{E}^{\text {pot }}$ with $\nabla \times \boldsymbol{E}^{\text {pot }}=0$. The conductances and $\boldsymbol{E}^{\text {pot }}$ may be arbitrary (yet physically reasonable) functions of time and position. In this paper we use empirical models from previous data-based studies. The output of the calculation method is the induced rotational part of the electric field, $\boldsymbol{E}^{\text {ind }}$ with $\nabla \cdot \boldsymbol{E}^{\text {ind }}=0$.

The potential electric field that we use as input may have been obtained from measurements or from MHD simulation by mapping the magnetospheric electric field down to the ionosphere along field lines. In principle, when we measure the ionospheric electric field we get the total field, including the induced rotational part. However, several standard analysis methods such as AMIE (Richmond and Kamide, 1988), KRM, (Kamide et al., 1981) or the SuperDARN potential mapping technique (Ruohoniemi and Baker, 1998) are based on the assumption that the ionospheric electric field is a potential field. This assumption is also used in many data-based ionospheric models (e.g. Untiedt and Baumjohann, 1993), as well as in ionospheric solvers of magnetospheric MHD simulations (e.g. Janhunen, 1998). The potential electric field obtained with any of the above techniques may be used as input in our calculation method, which then gives the induced rotational part of the ionospheric electric field as output.

The calculation method is based on Cartesian Elementary Current Systems (CECS), that were introduced by Amm (1997). There are two kinds of elementary systems, curl-free (CF) and divergence-free (DF), which form a set of basis functions for representing 2-dimensional vector fields. The general outline of the calculation method is following:

- Express the potential $\left(\boldsymbol{E}^{\mathrm{pot}}\right)$ and induced rotational $\left(\boldsymbol{E}^{\text {ind }}\right)$ parts of the electric field using CECS.

- $\boldsymbol{E}^{\text {pot }}$ is associated with a current system $\boldsymbol{j}_{1}$ and $\boldsymbol{E}^{\text {ind }}$ with $\boldsymbol{j}_{2}$. Express these currents with CECS and use Ohm's law to relate the CECS representations of $\boldsymbol{E}^{\text {pot }}$ and $\boldsymbol{j}_{1}$ as well as $\boldsymbol{E}^{\text {ind }}$ and $\boldsymbol{j}_{2}$. 


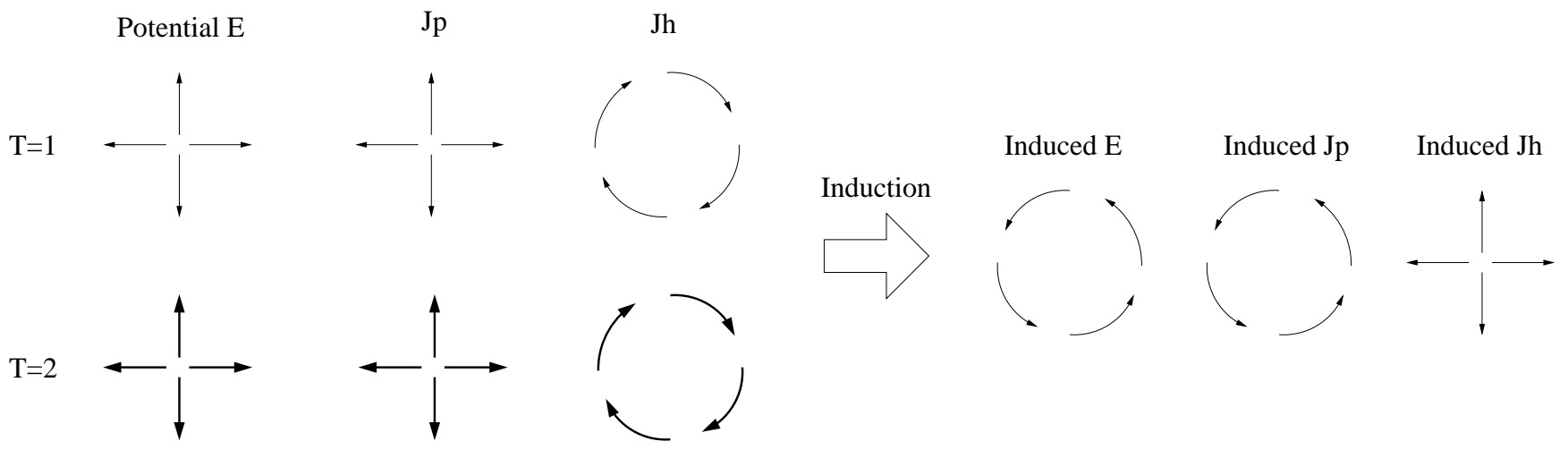

Fig. 1. Lenz's law in the northern auroral ionosphere (with uniform conductances and downward pointing background magnetic field). Changes of the potential electric field $\left(\boldsymbol{E}^{\text {pot }}\right)$ and associated currents (Jp and Jh) create rotational induced electric field $\left(\boldsymbol{E}^{\text {ind }}\right)$. Induced currents oppose the change in rotational currents, but enhance the change in divergent currents (i.e. FAC).

- Calculate the magnetic field $\boldsymbol{B}$ created by the currents.

- Faraday's law relates the unknown $\boldsymbol{E}^{\text {ind }}$ to the magnetic field $\boldsymbol{B}$.

In the CECS representation Ohm's law and Faraday's law give us a system of linear algebraic equations that relate the unknown scaling factors of the CECS representation of $\boldsymbol{E}^{\text {ind }}$ to the scaling factors of the input field $\boldsymbol{E}^{\text {pot }}$ and conductances $\Sigma_{H}, \Sigma_{P}$. The CF and DF CECS have been defined so that they have either a Dirac $\delta$-function curl or divergence at their center. This means that the calculation method is essentially a finite element method (FEM), where the basis functions (CECS) describe the curl and divergence of the electric field. The CECS basis functions are very convinient for the ionospheric induction problem, for they make it easy to divide electric fields and currents into curl- and divergencefree parts, which is essential in the calculations. Moreover, use of the CECS basis converts spatial differential equations into systems of linear algebraic equations, where boundary conditions are implicitely included. Detailed description of the method is given in Vanhamäki et al. (2006).

\section{Features of ionospheric induction}

\subsection{Lenz's law in the ionosphere}

Lenz's law states that the direction of the induced electric field in a loop of wire is such that the induced current opposes the change of the magnetic flux through the loop. Figure 1 is a schematic presentation of the ionospheric induction process. The potential electric field with associated Pedersen and Hall currents is on the left side. The potential E-field increases between $\mathrm{T}=1$ and $\mathrm{T}=2$ and the induced electric field and currents are on the right side. The inductive electric field is a rotational field, which (when conductances are uniform) is associated with a rotational Pedersen current and divergent
Hall current. The induced currents oppose the change in the rotational current, and hence also the change of magnetic flux through the ionospheric plane, but enhance the change in the divergent currents. This tendency of inductive currents to enhance the change of FAC was also noted by Buchert (1998) and Yoshikawa and Itonaga (2000).

The z-component of the electric field is assumed to be very small due to very high conductivity along the magnetic field, so the rotational $\boldsymbol{E}^{\text {ind }}$ is given by the $z$-component of Faraday's law,

$\left(\nabla \times \boldsymbol{E}^{\text {ind }}\right)_{z}=-\frac{\partial B_{z}}{\partial t}$.

In the CECS representation it is easy to see that only divergence-free currents are associated with the $\mathrm{z}$-component of the magnetic field (Vanhamäki et al., 2006, Eqs. 6-7). The induced electric field is also divergence-free, and according to Lenz's law it opposes the change in the divergence-free currents. Therefore we can estimate

$\boldsymbol{E}^{\text {ind }} \approx-\mu_{0} l \frac{\partial \boldsymbol{J}^{d f}}{\partial t}$,

where $l$ is a typical length scale and $\boldsymbol{J}^{d f}$ is the ionospheric divergence-free current. These kind of estimates, when applied to realistic data-dased models of different ionospheric current systems, are compared with exact results in Sect. 4.

\subsection{Dependence on ionospheric parameters}

Before studying some realistic, data-based models, let us first consider a simple example: the input potential electric field is rotationally symmetric and oscillates harmonically with frequency $\omega=2 \pi / T$, while ionospheric conductances are uniform. The input field $\boldsymbol{E}^{\text {pot }}$ is radial in cylindrical coordinates, with uniform sources at the origin, $\rho \leq 35 \mathrm{~km}$, and at an outer ring $115 \mathrm{~km} \leq \rho \leq 155 \mathrm{~km}$. The magnitudes of the sources are chosen so that the potential electric field outside 


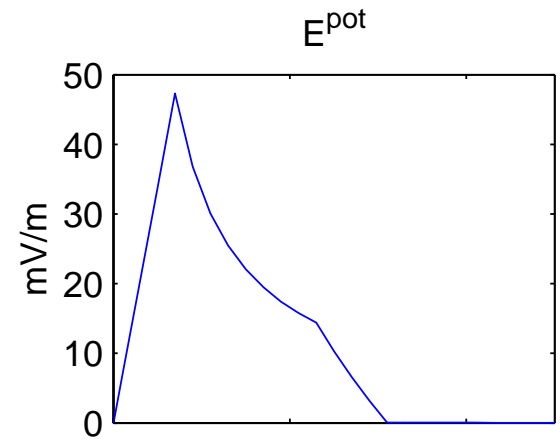

FAC1

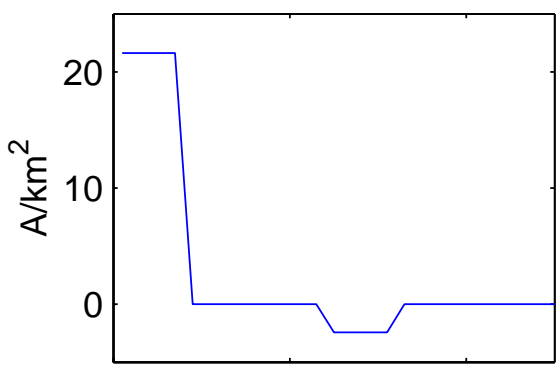

11

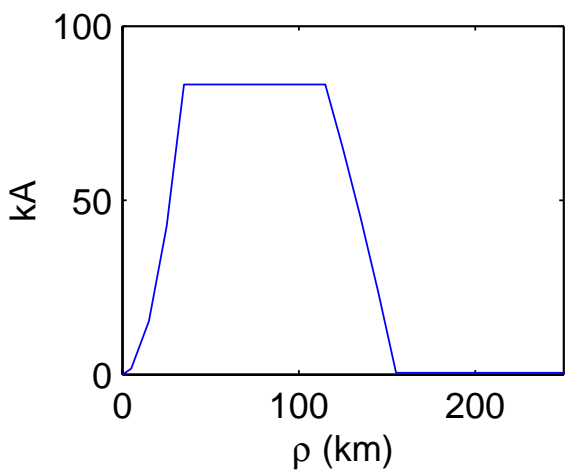

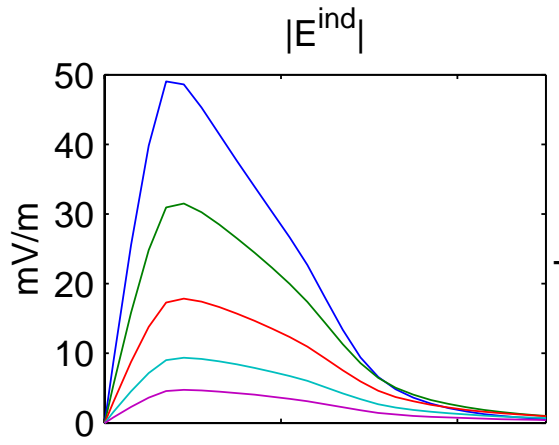

$\mid F A C^{\text {ind }}$
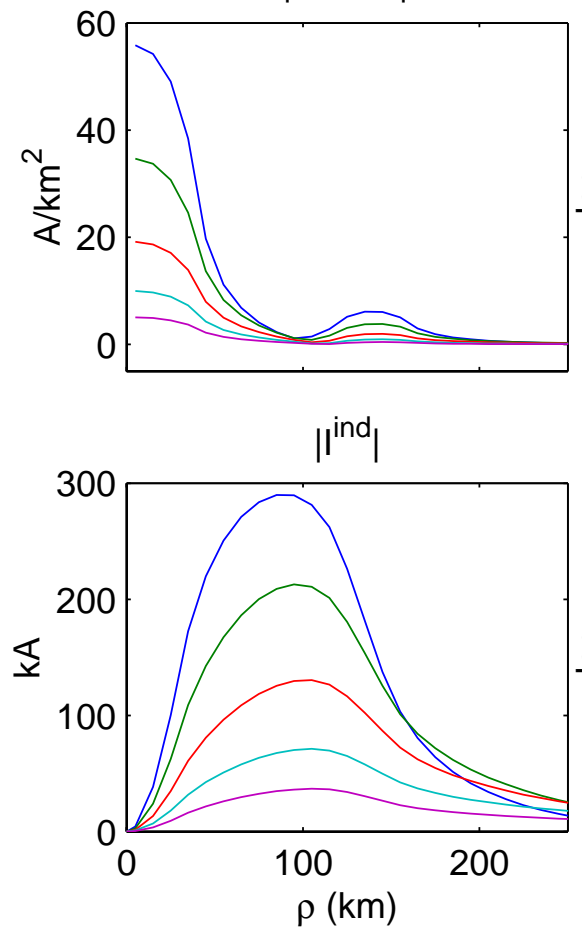
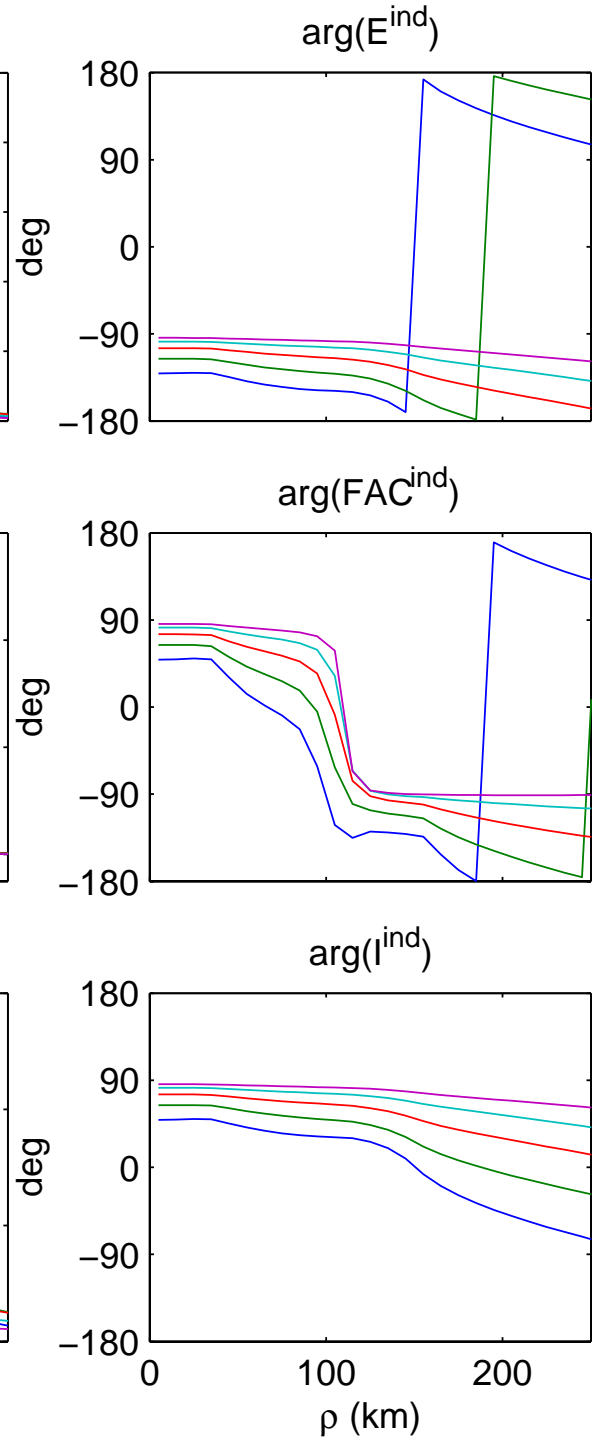

Fig. 2. Input system (electric field $\boldsymbol{E}^{\text {pot }}$, field-aligned current density FAC1 and the total field-aligned current I1) is rotationally symmetric and oscillates harmonically in time. Upper row: Input electric field, and magnitude and phase of the induced electric field $\boldsymbol{E}^{\text {ind }}$. Middle row: Same for the FAC. Bottom row: same for the integrated FAC inside radius $\rho$. In these examples conductances are uniform, $\Sigma_{P}=8 \mathrm{~S}$ and $\Sigma_{H}=16 \mathrm{~S}$. Oscillation time $T$ is varied: $T=1, T=2, T=4, T=8$ and $T=16$.

the radius $\rho=155 \mathrm{~km}$ is zero. This means that also the input current system is confined in this region. In the numerical calculations a rectangular $61 \times 61$ element grid with $10 \mathrm{~km}$ spacing was used. The situation with uniform conductances and harmonic time-dependence was discussed in Sect. 2.2 of Vanhamäki et al. (2006). According to their Eq. (15) the induced electric field depends on the ratios $\Sigma_{H} / T$ and $\Sigma_{P} / T$. Here we vary each of the parameters $T, \Sigma_{P}$ and $\Sigma_{H}$ one at a time, while keeping the other two fixed.

Figure 2 shows the input and induced electric field, FAC and integrated FAC for different oscillation times, between $T=1 \mathrm{~s}$ and $T=16 \mathrm{~s}$. In this example ionospheric conductances are $\Sigma_{P}=8 \mathrm{~S}$ and $\Sigma_{H}=16 \mathrm{~S}$. The top row of the figure shows the radial profiles of the input potential electric field and the magnitude and phase (with respect to the input field's $e^{i \omega t}$ ) of the induced rotational electric field for different $T$. The middle and bottom rows show the FAC and its integral

$I(\rho)=2 \pi \int_{0}^{\rho} \operatorname{FAC}\left(\rho^{\prime}\right) d \rho^{\prime}$,

respectively. Figure 3 shows in a similar manner the effects of different Pedersen conductances on the induced fields. In general the induced electric field is smoother and more broadly distributed than the input field. This is expected, for according to Eq. (1) induction is non-local in space, so that local changes in current systems create $\boldsymbol{E}^{\text {ind }}$ that extends 

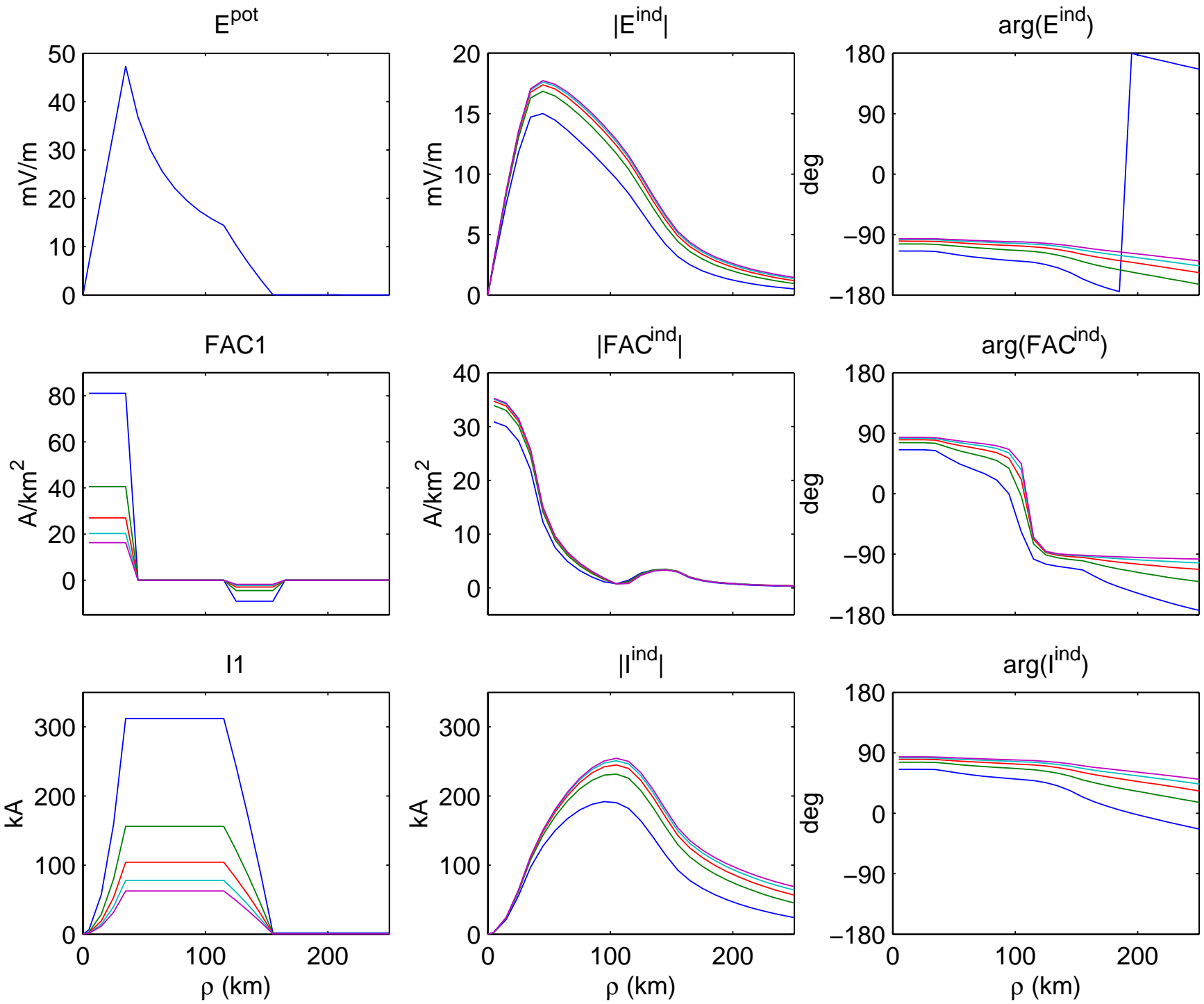

Fig. 3. Similar to Fig. 2, but now the Pedersen conductance $\Sigma_{P}$ is varied while keeping the oscillation time $T$ and Hall conductance $\Sigma_{H}$ constant. In these examples $T=8 \mathrm{~s}$ and $\Sigma_{H}=30 \mathrm{~S}$, while the Pedersen conductance is $\Sigma_{P}=30, \Sigma_{P}=15, \Sigma_{P}=10, \Sigma_{P}=7.5$ and $\Sigma_{P}=6$.

outside the area of changing currents. The induced electric field is also roughly 90 degrees behind the input field, at least near the origin, which is related to the fact that $\boldsymbol{E}^{\text {ind }}$ depends on the time derivative of the magnetic field.

The effect of different oscillation times on the induced fields is clearly visible in Fig. 2. With the shortest oscillation time $T=1 \mathrm{~s}$ the induced field $\boldsymbol{E}^{\text {ind }}$ has a peak magnitude similar to the input potential field, while with $T=16 \mathrm{~s}$ the induced field is already quite small. When $T>4 \mathrm{~s}$ the magnitude of the induced electric field depends almost linearly on the frequency $\omega=2 \pi / T$. However, with smaller $T$ the induced field increases less rapidly with $\omega$ and is also more localized, decreasing faster at large distances. The FAC, shown in the middle row of Fig. 2, are concentrated near the origin and at the outer ring, where the sources of the potential electric field are located. The induced FAC are spread in a wider area than those of the input system and the $\sim 90$ degree phase difference means that the induced FAC is enhancing the change of the input FAC. However, the phase difference deviates notably from 90 degrees with small $T$. The total current flowing in the field aligned direction inside a given radius is given by $I$, shown in the lower row of Fig. 2. The input FAC system is exactly balanced, so that equal amounts of FAC flow into the ionosphere at $\rho \leq 35 \mathrm{~km}$ and out of it at $115 \mathrm{~km} \leq \rho \leq 155 \mathrm{~km}$. Also the induced upward and downward FAC are nearly balanced. The increasingly localized nature of the induced system with smaller $T$ is clearly visible in the $\left|I^{\text {ind }}\right|$ profiles. It seems that the ionospheric induction process affects the FAC more than the electric field, for the induced FAC are much larger 

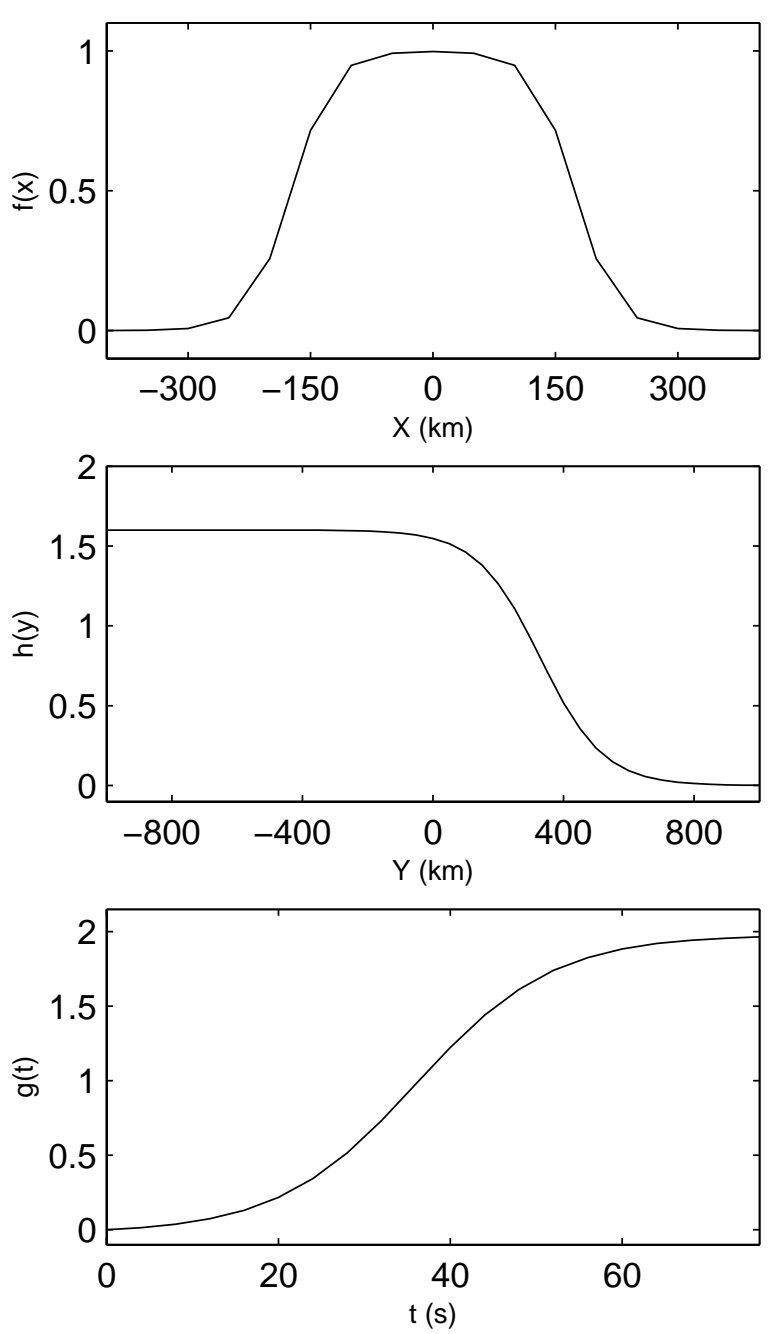
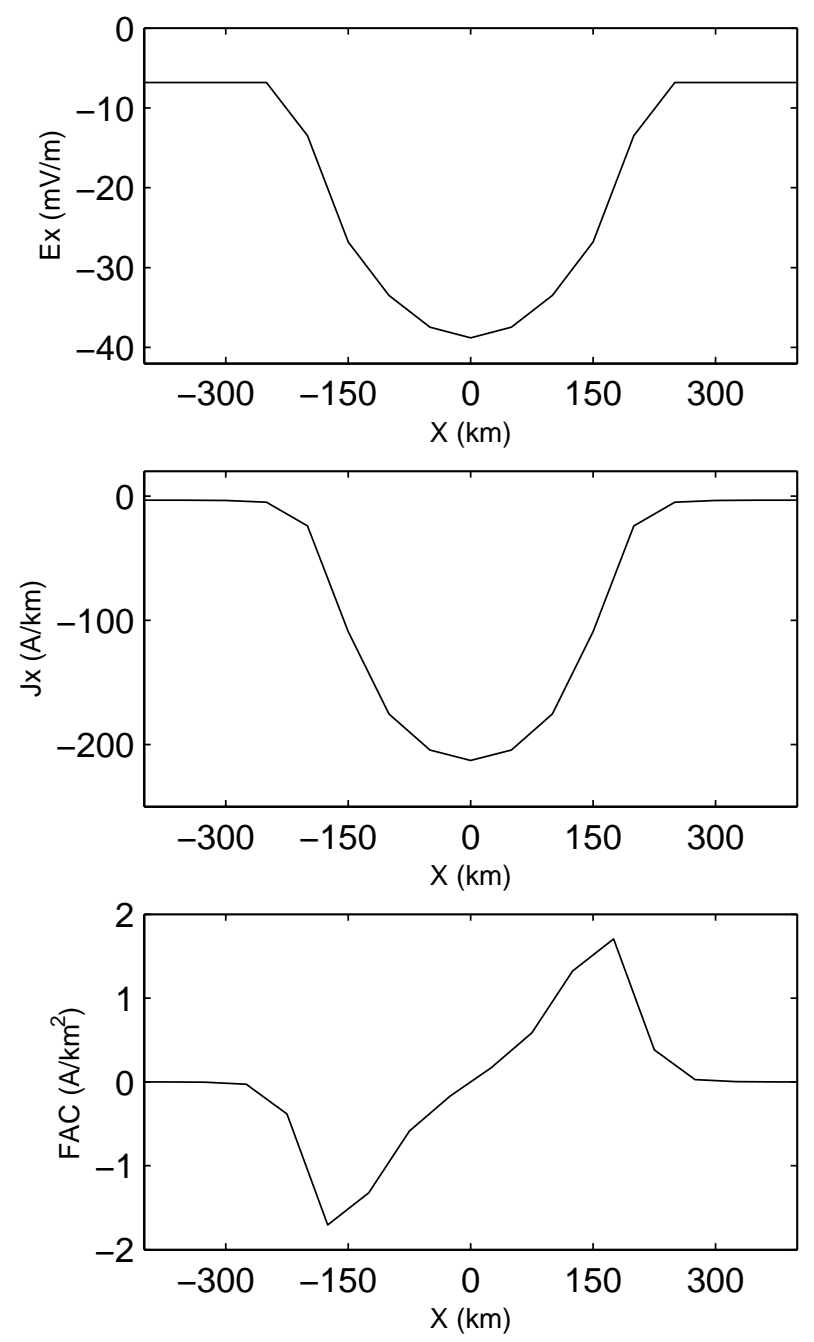

Fig. 4. On the left side the functions $f(x), h(y)$ and $g(t)$ giving the spatial and temporal dependences of the electrojet model (Eq. 4). On the right side the cross sectional shape of the North-South directed electric field and current together with FAC.

than the input FAC, although $\boldsymbol{E}^{\text {ind }}$ is at most equal to input $\boldsymbol{E}^{\text {pot }}$. This is partly explained by the larger spatial scale of the induced E-field and also by the ratio $\Sigma_{H} / \Sigma_{P}=2$ of the conductances. In the input system rotational currents are Hall currents and FAC are associated with Pedersen currents. In the induced system this is reversed, which means that $\max \left(\mathrm{FAC}^{\text {ind }} / \mathrm{FAC} 1\right) \propto 2 \max \left(\left|\boldsymbol{E}^{\text {ind }}\right| /\left|\boldsymbol{E}^{\mathrm{pot}}\right|\right)$.

Figure 3 shows the effect of varying Pedersen conductance $\Sigma_{P}$, while keeping the oscillation time $T$ and Hall conductance $\Sigma_{H}$ constant. The peak magnitude of the induced electric field increases slightly with decreasing $\Sigma_{P}$, and the field also decays somewhat slower with distance. This is related to the behaviour of the induced rotational currents, which are Pedersen currents in the case of uniform conductances. Induction opposes the change in the z-component of the magnetic field, which in turn depends on the rotational currents. Thus induced rotational currents, that get stronger with $\Sigma_{P}$, "screen" the input system, and make induced E-field slightly smaller and more localized. The induced FAC depend on
Hall conductivity and on the derivative of the induced electric field in the radial direction. Therefore changing $\Sigma_{P}$ does not affect induced FAC significantly, although the input FAC are varied linearly with $\Sigma_{P}$.

In contrast to the non-trivial $T$ and $\Sigma_{P}$ dependences, Eq. (15) of Vanhamäki et al. (2006) shows that the induced electric field depends linearly on Hall conductance. This is reasonable, for induction depends on the temporal changes of the z-component of the magnetic field, as explained in the previous section. $B_{z}$ on the other hand is associated with rotational currents. In the case of uniform conductances, rotational currents of the input system are Hall currents, while rotational currents of the induced system are Pedersen currents. Therefore increasing $\Sigma_{H}$ has the same effect on induction as increasing the strength of the input electric field by some constant factor. Consequently $\boldsymbol{E}^{\text {ind }}$ depends linearly on $\Sigma_{H}$, and the induced FAC, which are Hall currents, depend on $\Sigma_{H}^{2}$. 

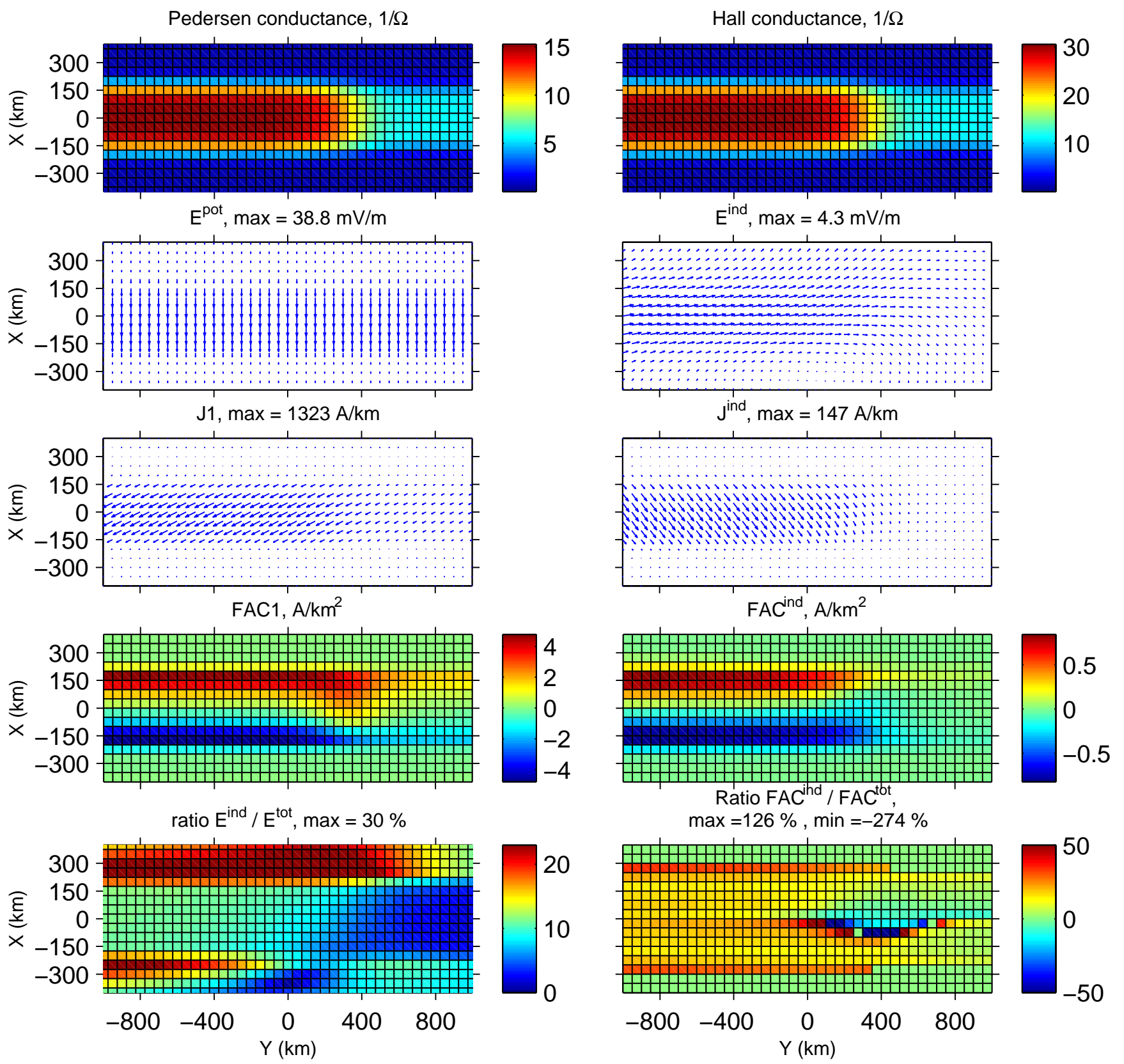

Fig. 5. The input electrojet model (Pedersen and Hall conductances, potential electric field $\boldsymbol{E}^{\text {pot }}$ with current system $\boldsymbol{J} \mathbf{1}$ and FAC1) and the calculated induced electric field $\left(\boldsymbol{E}^{\text {ind }}\right)$ and current system $\left(\boldsymbol{J}^{\text {ind }}\right.$ and FAC $\left.{ }^{\text {ind }}\right)$ at time instant $t=40 \mathrm{~s}$. Comparison between the induced and total (input + induced) electric field and FAC are also shown.

\section{Inductive fields in different ionospheric systems}

Next we present results for three realistic ionospheric situations, namely a non-uniform electrojet, WTS and $\Omega$-band. The electrojet model is based on models presented by Untiedt and Baumjohann (1993) and Amm (1995). The WTS and $\Omega$ band models have been published by Amm (1995) and Amm (1996). They are constructed from observational data obtained at northern Scandinavia by the Scandinavian Magne- tometer Array, EISCAT radar and magnetometer cross, and STARE radar.

\subsection{Non-uniform electrojet}

Our first example is an electrojet that intensifies and becomes more non-uniform with time. The input model is shown in Figs. 4 and 5. The electrojet flows in the y-direction and the input electric field $\boldsymbol{E}^{\text {pot }}$ is constant in time and uniform in 

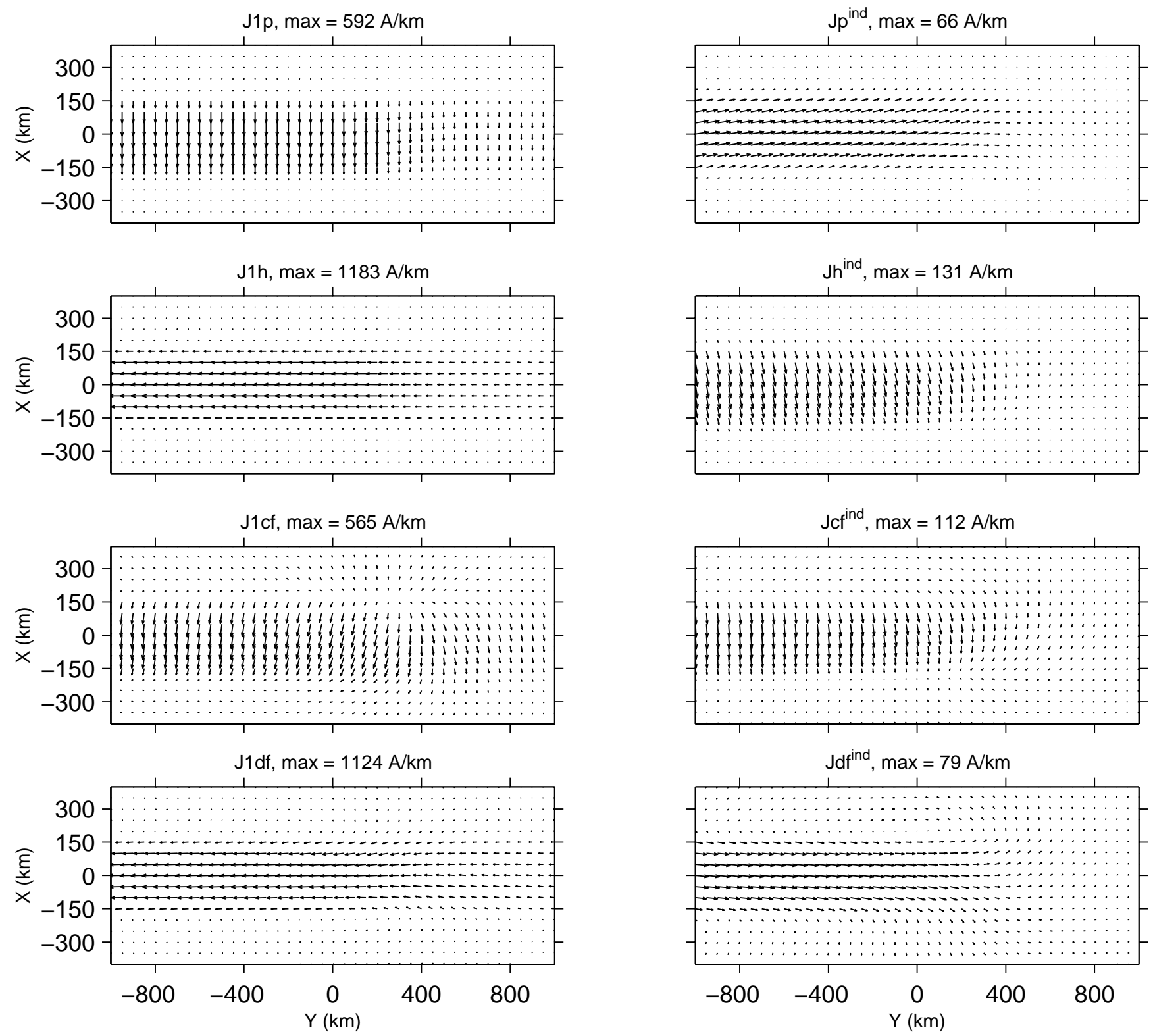

Fig. 6. The division of original (1) and induced (ind) currents of the electrojet model into Pedersen (Jp), Hall (Jh), curl-free (Jcf) and divergence-free (Jdf) parts at time instant $t=40 \mathrm{~s}$

the y-direction. The cross section of $\boldsymbol{E}^{\text {pot }}$ over the electrojet is shown in the upper right panel of Fig. 4. The time variations and y-dependence of the model are in the conductances, which are defined as

$\Sigma_{P}=\Sigma_{H} / 2$

$\Sigma_{H}=1+f(x)[1+h(y) g(t)]$.

Functions $f(x), h(y)$ and $g(t)$ are given in the left side panels of Fig. 4. The calculation area where the CECS representing the induced electric field are placed is $-625 \mathrm{~km} \leq x \leq 625 \mathrm{~km}$ by $-3025 \mathrm{~km} \leq y \leq 3025 \mathrm{~km}$, with
$50 \mathrm{~km}$ resolution in both directions. In the beginning the conductivity distributions are uniform in the $y$-direction. Cross sectional profiles of the resulting $J_{x}$ and FAC distributions are shown in middle and lower right panels of Fig. 4. In the western part of the electrojet the conductances begin to increase, thus intensifing the current, while the eastern part of the electrojet is constant. The electrojet becomes nonuniform, so that the transition region between the weaker and stronger jet is in the area $0 \mathrm{~km}<y<600 \mathrm{~km}$. Largest time derivatives occur at $t=40 \mathrm{~s}$, at which point the time derivative of the ground magnetic field is $\sim 13 \mathrm{nT} / \mathrm{s}$ below the center of jet. This time derivative is quite high, but still reasonable, as 

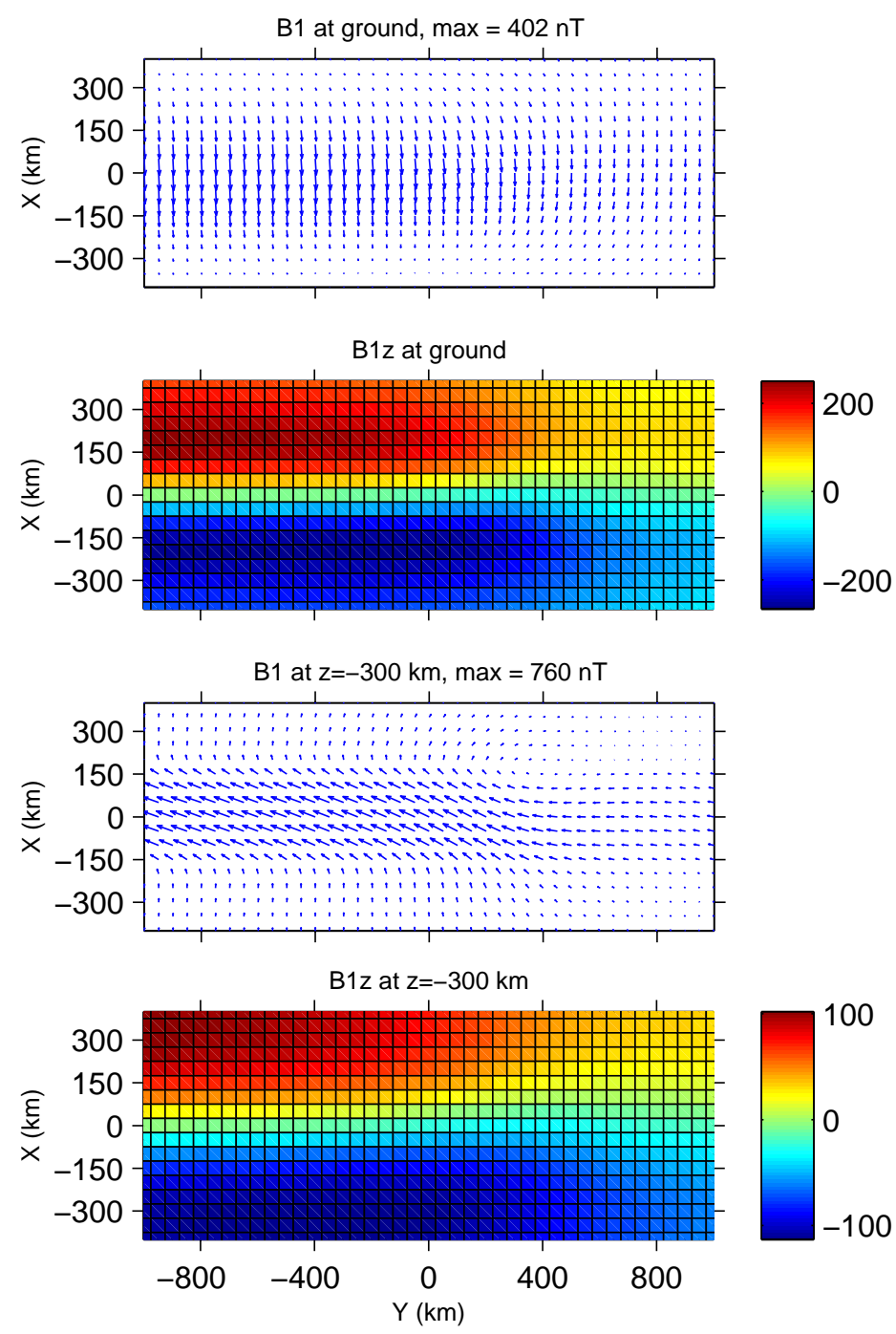
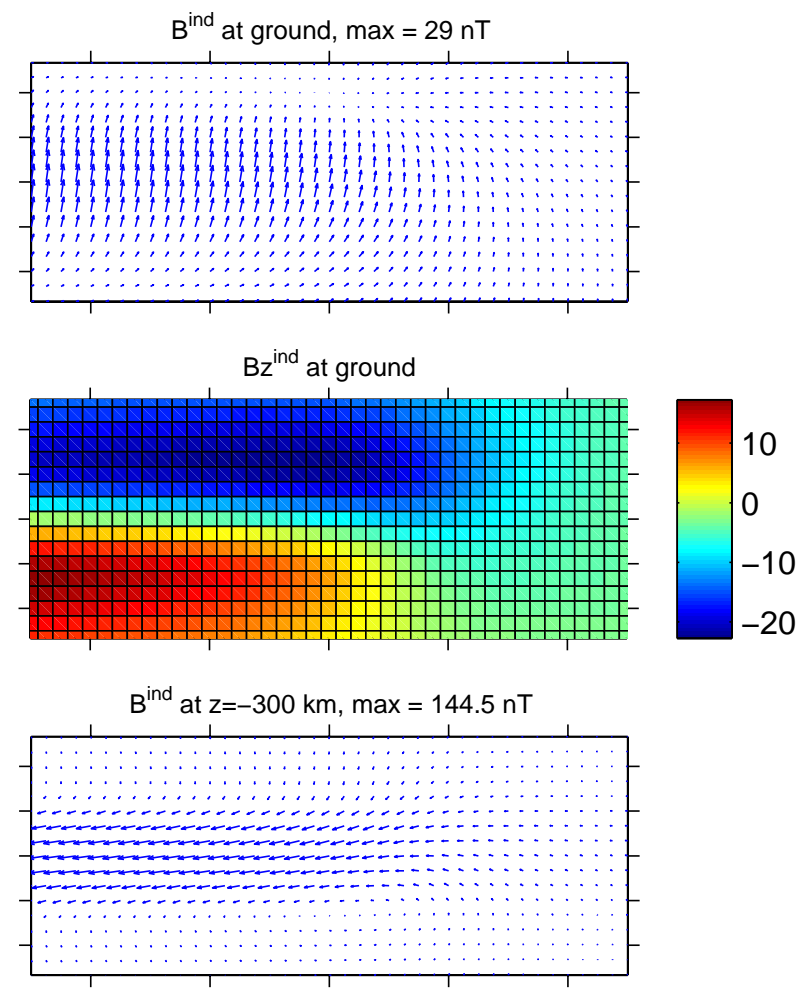

$\mathrm{Bz}^{\mathrm{ind}}$ at $\mathrm{z}=-300 \mathrm{~km}$

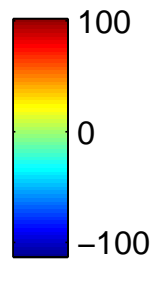

Fig. 7. The magnetic fields associated with the input $(\boldsymbol{B 1})$ and induced $\left(\boldsymbol{B}^{\text {ind }}\right)$ electrojet system at the ground and at $300 \mathrm{~km}$ above the ionospheric current layer at time instant $t=40 \mathrm{~s}$.

these kind of values have been observed during large storms (Pulkkinen et al., 2005).

The input and induced systems at $t=40 \mathrm{~s}$ are illustrated in Figs. 5-7. The input electrojet consists of a southward electric field and South-West currents that are concentrated in the $\mathrm{x}$-direction to a $\sim 300 \mathrm{~km}$ wide channel, where also the conductances are enhanced. FAC are focused on two narrow bands at the northern and southern edges of the electrojet, except at the transition region where a downward FAC feed the intensifying ionospheric current. The induced electric field $\boldsymbol{E}^{\text {ind }}$ is directed eastward and is quite uniform in the area where the input electrojet increases. In this area the induced E-field is about $10 \%$ of the input field. The induced E-field is not symmetric in the North-South direction, but spreads farther out in the North. This is clearly visible in the lower left panel of Fig. 5, where the ratio $\left|\boldsymbol{E}^{\text {ind }}\right| /\left|\boldsymbol{E}^{\text {pot }}+\boldsymbol{E}^{\text {ind }}\right|$ is illustrated.
Table 1. Dependence of $\max \left(\left|\boldsymbol{E}^{\text {ind }}\right|\right)$ on the duration $T$ of the electrojet intensification. In these examples $T$ is changed by a factor of 2 while keeping all other parameters fixed. Also the ratio between the maximum values of $\boldsymbol{E}^{\text {ind }}$ for different $T$ is given.

\begin{tabular}{ccccccc}
\hline$T$ & 19 & 38 & 76 & 152 & $304 \mathrm{~s}$ \\
$\max \left(\left|\boldsymbol{E}^{\text {ind }}\right|\right)$ & 12.31 & 7.74 & 4.30 & 2.24 & $1.14 \mathrm{mV}$ \\
Ratio & \multicolumn{2}{c}{1.59} & 1.80 & 1.92 & 1.96 & \\
\hline
\end{tabular}

The induced currents, shown in the middle right panel of Fig. 5, flow in the South-East direction. They oppose the change of the main westward electrojet, but increase the change in southward currents and consequently in the FAC at northern and southern edges of the jet. In the input system there is an excess of downward currents, the total amount 


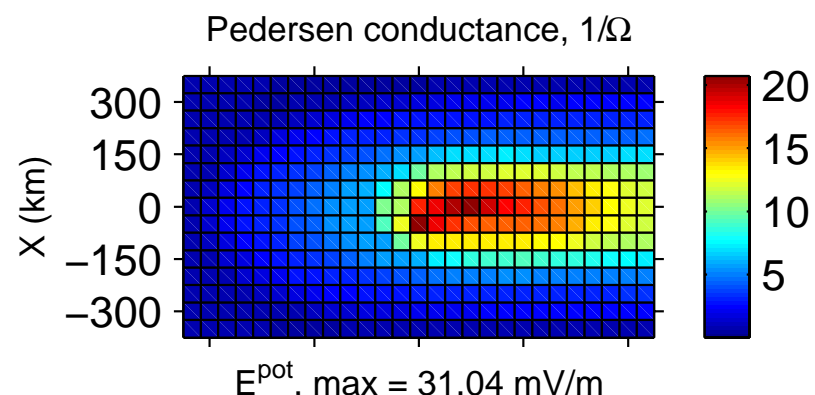

Hall conductance, $1 / \Omega$

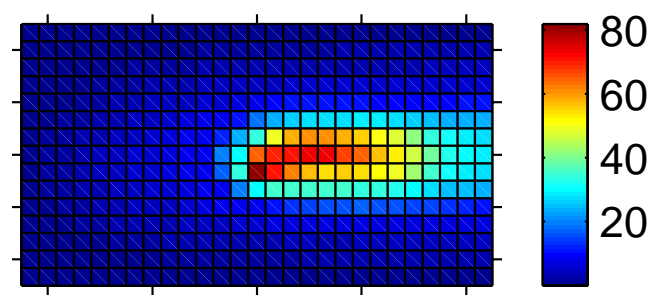

$E^{\text {ind }}, \max =1.97 \mathrm{mV} / \mathrm{m}$
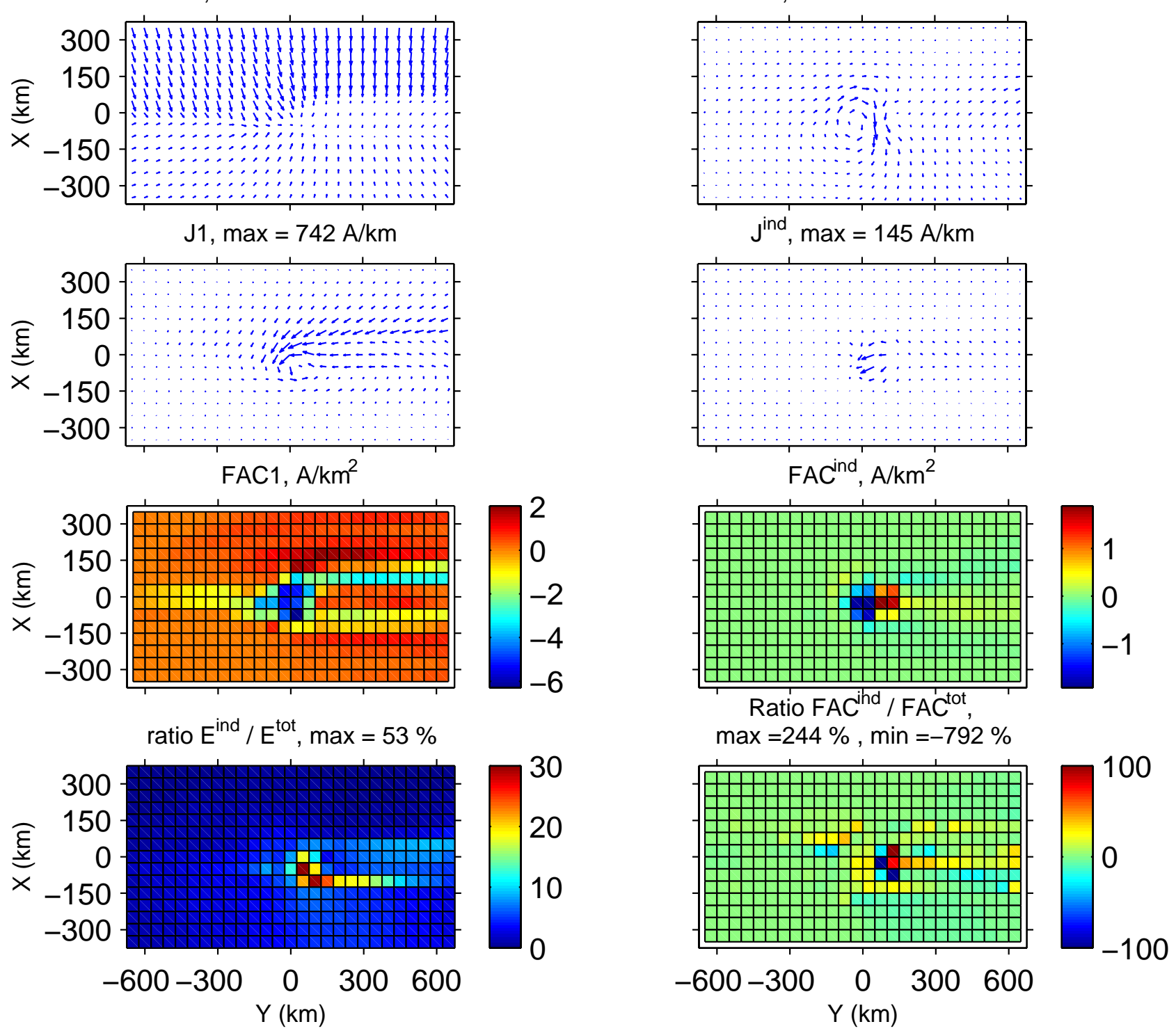

Fig. 8. Same as Fig. 5, but for the WTS model.

of upward FAC being $\sim 80 \%$ of the downward FAC. This excess downward FAC feeds the intensifying western part of the electrojet. In the induced system there is $\sim 15 \%$ imbalance in the opposite direction, whith the excess induced FAC flowing upwards in the transition region between the weaker and stronger jet areas. The ratio $\left|J^{\text {ind }}\right| /\left|J^{\text {tot }}\right|$ is just the same as for the electric field, $\sim 10 \%$ in the area of the enhanced electrojet. However, the induced FAC contribute about $20 \%$ of the total FAC of the enhanced electrojet, as is visible in the lower right panel of Fig. 5. The division of 

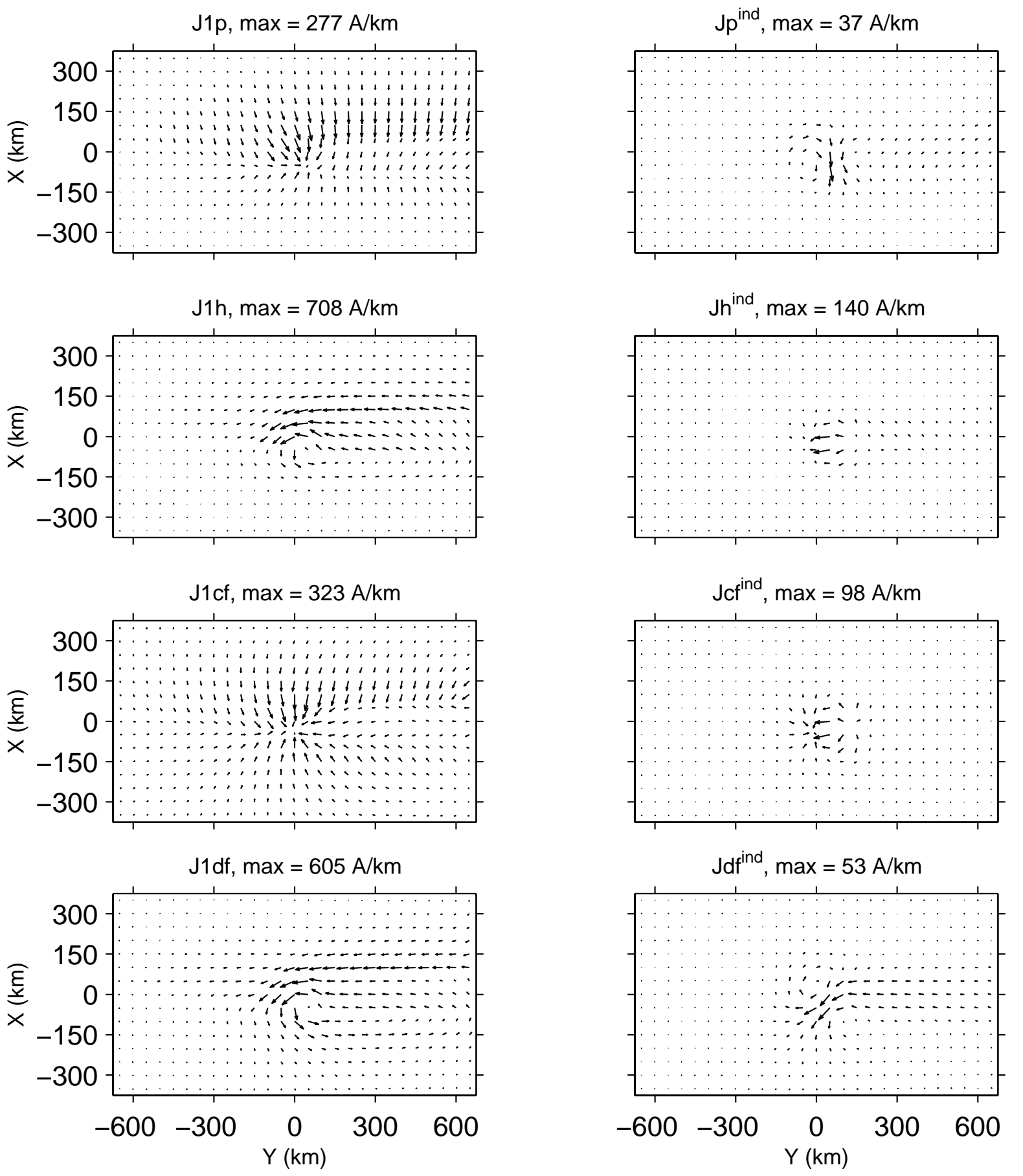

Fig. 9. Same as Fig. 6, but for the WTS model.

the input and induced current systems into Pedersen, Hall, curl-free and divergence-free parts is given in Fig. 6. The induced currents oppose the change of divergence-free currents but increase the change of curl-free currents and associated FAC. This behaviour is in accordance with Lenz's law, as explained in Sect. 3. Most of the FAC in the input model are associated with Pedersen currents, but in the induced system
FAC are mostly connected to Hall currents. This is expected, for in the input system electric field is irrotational, but the induced electric field is divergence-free. As the Hall conductance is larger than Pedersen conductance, this behaviour also explains why (at least in this example) induction seems to affect FAC more than the horizontal currents or electric field. The Pedersen and Hall currents of the input system 

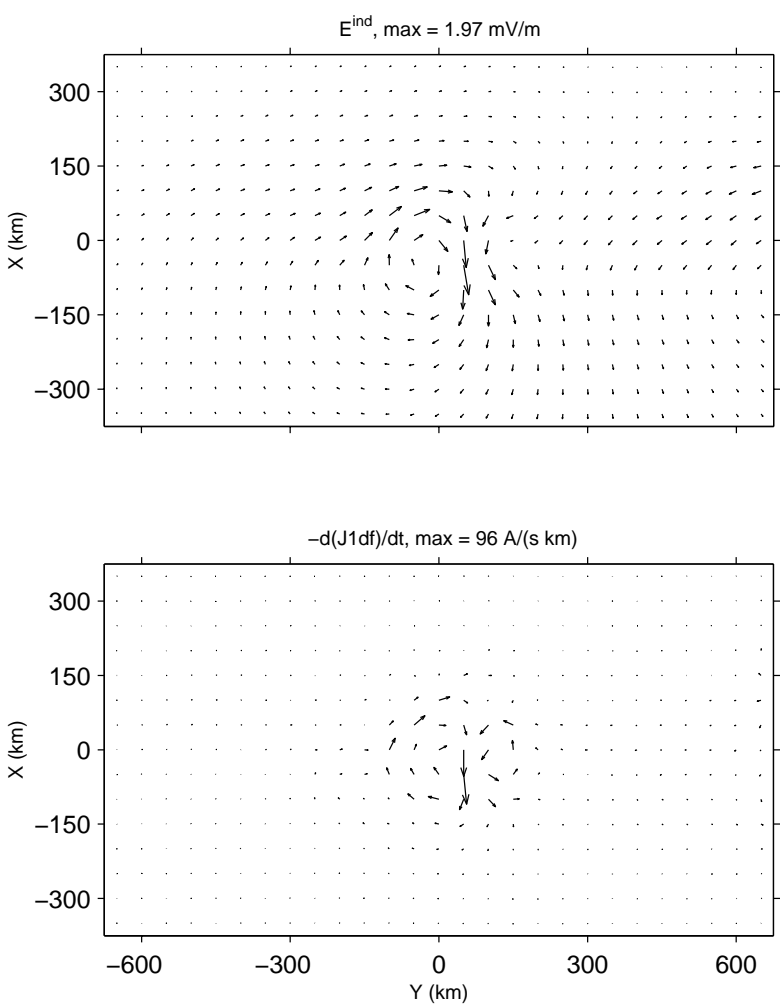

Fig. 10. Comparison between the induced electric field (same as in Fig. 8) and negative time derivative of the divergence-free currents of the input system.

are symmetric in the North-South direction, but the curl- and divergence-free currents are unsymmetric because of the extra FAC flowing at the transition region. The slight asymmetry of the divergence-free currents is the reason why the induced electric field is also asymmetric, as noted above.

The magnetic fields associated with the input and induced current systems are illustrated in Fig. 7 at two different altitudes, at ground and $300 \mathrm{~km}$ above the ionospheric current layer. At ground level the induced magnetic field opposes the change of the original field. The magnitude of the induced B-field is $\sim 5 \%$ in the horizontal part and $\sim 10 \%$ in the vertical part when compared to the original field. The asymmetry of the induced system is clearly visible, especially in the vertical component. Above the ionosphere the induced $B_{z}$ is opposite to the original field, but the horizontal component is rotated by almost 90 degrees. The magnitude of the induced $B_{z}$ is again about $10 \%$, but in the horizontal component the magnitude of the induced field is over $15 \%$ of the original field. This larger contribution is mainly due to the induced FAC, that contribute to the horizontal magnetic field above the ionosphere.

In this example the intensification of the electrojet took place in $T=76 \mathrm{~s}$, as shown in the lower left panel of Fig. 4. In the numerical calculations this time interval was divided into 20 steps with $\Delta t=4 \mathrm{~s}$. Table 1 shows the peak magni- tudes of the induced electric field for different values of $T$. In these examples only the total duration $T$ of the electrojet intensification is changed while keeping the form of temporal variations and other parameters the same. Changing $T$ from $304 \mathrm{~s}$ to $152 \mathrm{~s}$ doubles the magnitude of the induced electric field, as expected when all time derivatives are doubled. However, further decreases in $T$ result in smaller increases in the induced field. The induction process becomes non-linear as the induced currents reach values that are comparable to the input system. When the intensification happens in time span of $T=19 \mathrm{~s}$ the magnitude of the induced electric field is over $30 \%$ of the input potential field. This would mean that the maximum time derivative of the ground magnetic field is $\sim 51 \mathrm{nT} / \mathrm{s}$, which is an exceptionally high, yet observed value (Pulkkinen et al., 2005). Interestingly, changes in the size (width) of the electrojet have exactly the same effect on the induced electric field as changes in the temporal variations. It seems that the magnitude of the induced field depends (non-linearly) on the ratio $l / \tau$, where $l$ and $\tau$ are characteristic spatial and temporal scales, respectively. A similar result was obtained by Yoshikawa and Itonaga (1996) for the case of Alfvén wave reflection from ionosphere, while Buchert (1998) found dependence $l^{2} / \tau$.

\subsection{WTS}

The upper and left side panels of Fig. 8 show the input WTS model, Pedersen and Hall conductances $\Sigma_{P}$ and $\Sigma_{H}$, potential electric field $\boldsymbol{E}^{\text {pot }}$ and associated currents and FAC. The calculation area where the CECS representing the induced electric field are placed is $-625 \mathrm{~km} \leq x \leq 625 \mathrm{~km}$ by $-1025 \mathrm{~km} \leq y \leq 1425 \mathrm{~km}$, with $50 \mathrm{~km}$ resolution in both directions. Temporal variations are created by moving the whole system westward at $10 \mathrm{~km} / \mathrm{s}$, which is quite high but still a realistic speed (Paschmann et al., 2002, chapter 6). The maximum time derivative of the ground magnetic field in this case is $\sim 5 \mathrm{nT} / \mathrm{s}$. The induced electric field $\boldsymbol{E}^{\text {ind }}$ and currents $J^{\text {ind }}$ together with FAC are shown on the right side panels. The bottom panels of Fig. 8 show comparison of the induced E-field against the total field $\boldsymbol{E}^{\text {pot }}+\boldsymbol{E}^{\text {ind }}$ and induced FAC agains the total FAC.

The induced electric field $\boldsymbol{E}^{\text {ind }}$ in the WTS system is concentrated in a small area around the surge "head". The magnitude of the induced electric field is rather small compared to the largest potential field values present in the WTS. However, around the surge head the potential field is supressed due to large conductivity and the induced field contributes $20-50 \%$ of the total electric field in this small area. Previously Lühr et al. (1998) speculated that there might be induced electric fields of this order of magnitude in the WTS system, although they were not able to confirm this, as they used only single point measurements. Because the conductances are enhanced around the surge head, the induced current driven by $\boldsymbol{E}^{\text {ind }}$ is relatively large and the induced FAC are comparable to the original ones. Induced currents form a 

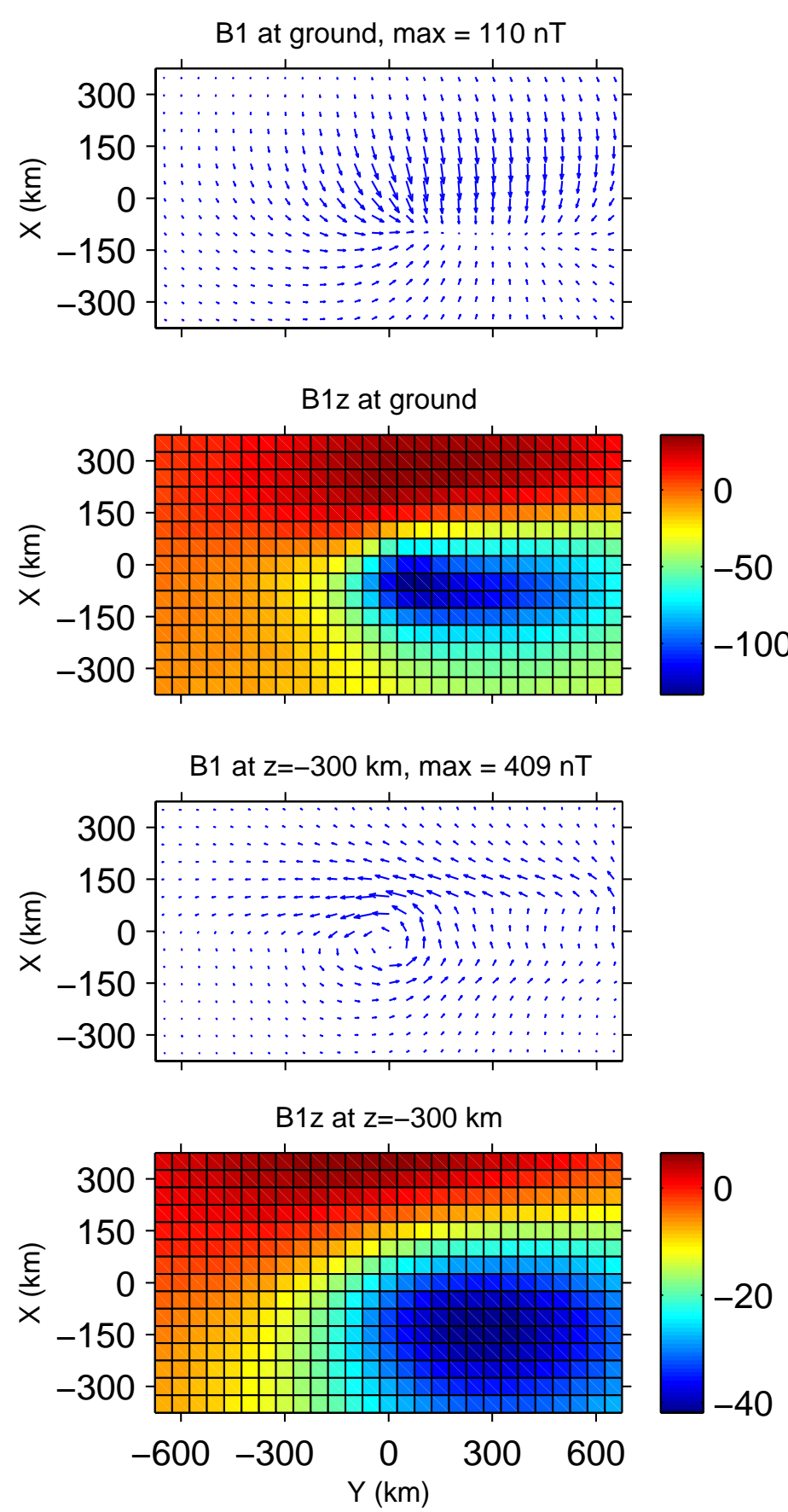
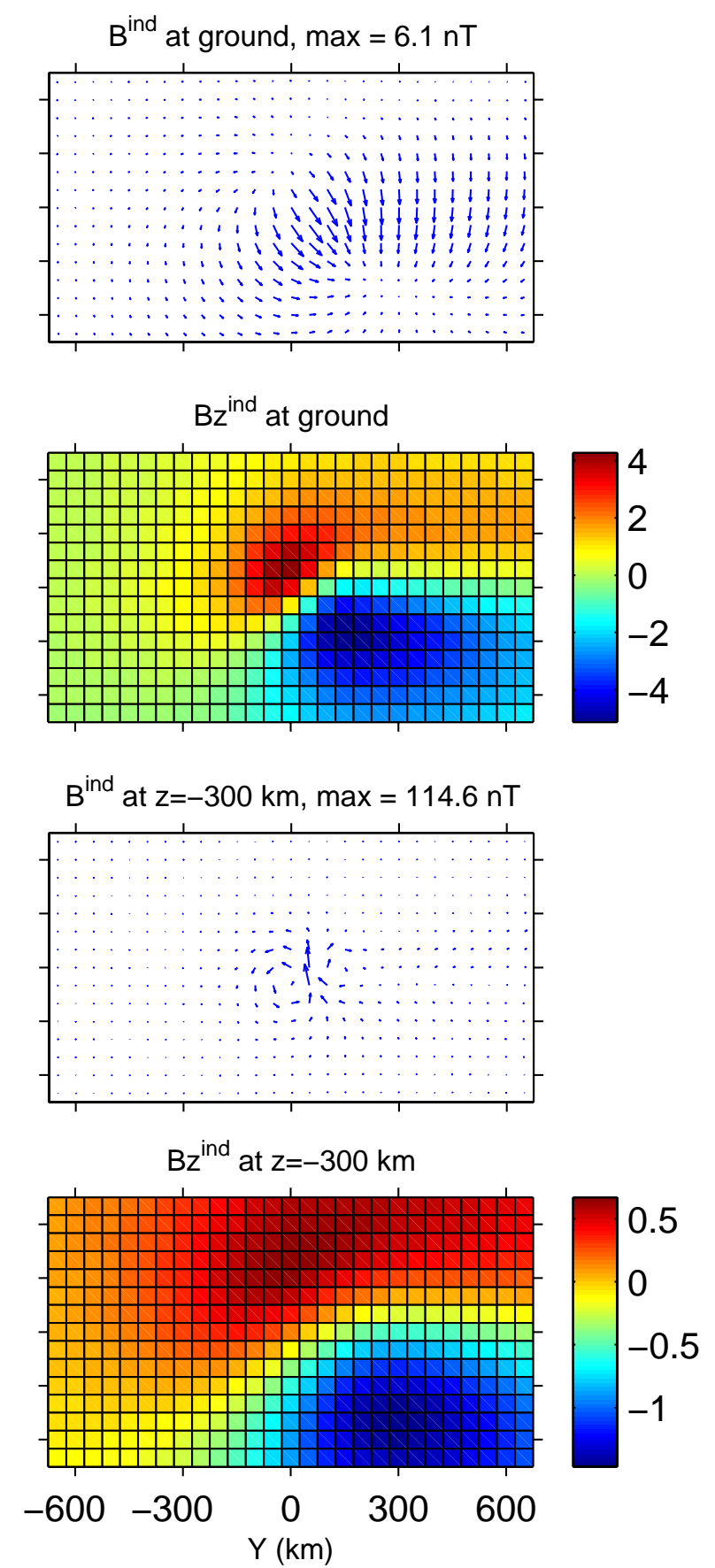

Fig. 11. Same as Fig. 7, but for the WTS model.

loop, with downward FAC at the eastern and upward FAC at the western part of the surge head. The induced FAC are almost balanced with a slight upward net current, the integrated downward current over the analysis region being $~ 90 \%$ of the upward current in the same area. In the input model the imbalance is larger, the upward FAC being 30\% larger than the total downward FAC. It is interesting to note that while the induced upward FAC are concentrated at the same areas where the FAC of the input system are largest, the induced downward FAC are located east of this area. This means that inductive processes do modify the nature of ionospheremagnetosphere coupling, at least in the WTS.

If the induced electric field of Fig. 8 is compared to the results of previous, more approximate results of Vanhamäki et al. (2005) (their Fig. 5), some similarities and differences are noted. First, both calculations give the induced electric field 


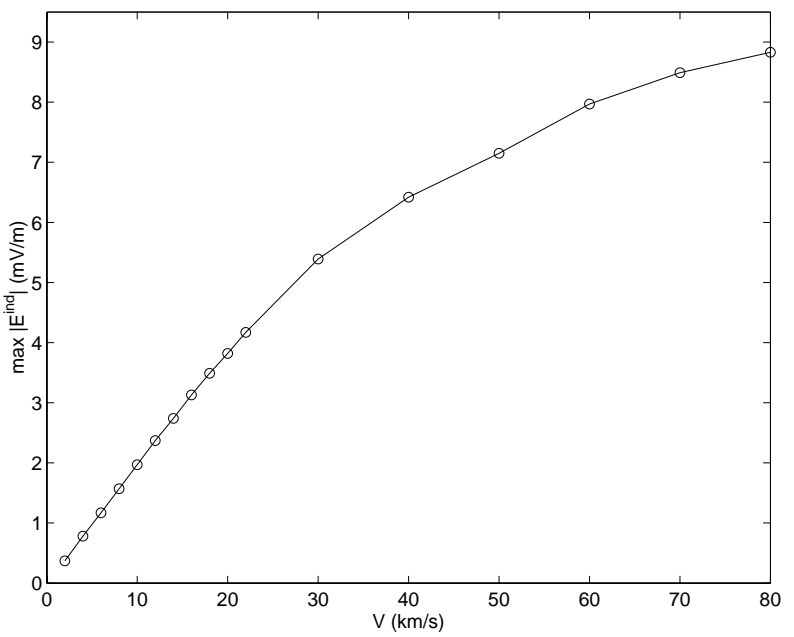

Fig. 12. The peak magnitude of the induced electric field for different velocities of the WTS system.

about the same magnitude $(1.97$ and $2.12 \mathrm{mV} / \mathrm{m})$ and in both cases the induced field is concentrated in the same area at the surge head. However, the orientation and spatial structure of the induced field obtained by Vanhamäki et al. (2005) is quite different from that found in this study. There are probably two reasons for the differences: Vanhamäki et al. (2005) calculated the induced field as a vacuum field (i.e. the second order effect of the induced current was ignored), and in their calculation method the induced field is not completely divergence-free.

Figure 9 shows the division of the input and induced currents into different parts. In the previous electrojet example there was a high degree of similarity between the induced Pedersen and divergence-free currents and also between induced Hall and curl-free currents. In the WTS case the similarity is not as prominent, although the induced Hall and curl-free currents have very much the same shape if not magnitude. This is probably due to the larger and sharper conductivity gradient that are present near the surge head, where the induced electric field and currents are concentrated. Indeed, in the input system, which is spread out in a larger area, there is still a close connection between the Pedersen and curl-free currents as well as between Hall and divergencefree currents.

In the electrojet example it was easy to see that the induced electric field opposes the change in the divergence-free part of the input system. In the WTS example this is more difficult to see immediately from Fig. 9 due to the spatial movement of the system. Figure 10 shows a direct comparison between the induced electric field and the time derivative of the input divergence-free current. There is indeed a close resemblance between the two vector fields, in accordance to Lenz's law as explained in Sect. 3. According to Fig. 10 typical length scale of $d\left(J 1^{d f}\right) / d t$ seems to be about $100 \mathrm{~km}$. Using this length scale in Eq. (2) gives an estimate for the magnitude of the induced electric field as $\left|\boldsymbol{E}^{\text {ind }}\right| \approx 12 \mathrm{mV} / \mathrm{m}$, which is too high by a factor of 6 .

Magnetic fields of the input and induced systems are shown in Fig. 11. The induced magnetic field at ground is almost negligible, less than $5 \%$ of the input field. Above the ionosphere, however, the horizontal part of $\boldsymbol{B}^{\text {ind }}$ is quite large, about $30 \%$ of the input field near the surge head. Horizontal magnetic field above the ionosphere is dominated by FAC, which explains the large contribution of the induced field.

Figure 12 shows how the peak magnitude of the induced electric field varies with the speed of WTS, with other parameters kept fixed. For all realistic speeds the dependence is linear. Only for speeds $>30 \mathrm{~km} / \mathrm{s}$ the induction process becomes non-linear, meaning that the induced currents themselves produce a significant $B_{z}$, that affects induction via Eq. (1). Also with these higher speeds the shape of the induced E-field is similar to $\boldsymbol{E}^{\text {ind }}$ in Fig. 8, although the induced field spreads over a larger area.

\section{$4.3 \Omega$-band}

Our third example is an ionospheric $\Omega$-band. The input model and the induced electric field and current are given in Figs. 13-14. The calculation area where the CECS representing the induced electric field are placed is $-625 \mathrm{~km} \leq x \leq 675 \mathrm{~km}$ by $-625 \mathrm{~km} \leq y \leq 1075 \mathrm{~km}$, with $50 \mathrm{~km}$ resolution in both directions. The input model is moving eastward at $2 \mathrm{~km} / \mathrm{s}$, which is again in the upper range of realistic speeds in terrestrial applications. In this case the time derivative of the ground magnetic field is about $5 \mathrm{nT} / \mathrm{s}$.

In the case of the $\Omega$-band the induced electric field $\boldsymbol{E}^{\text {ind }}$ shown in Fig. 13 is very similar to that obtained by Vanhamäki et al. (2005) using a more approximate calculation method. The peak value of the induced field is very small compared to the largest values of the potential field present in the input model. However, the largest values of $\boldsymbol{E}^{\text {ind }}$ occur in the $\Omega$ itself, i.e. in the area of enhanced conductivity, where the potential electric field is supressed. In this limited area the inductive part contributes up to $25 \%$ of the total electric field.

The induced downward FAC are concentrated at the western edge of the $\Omega$ and reaches values $\sim 20 \%$ of the input FAC, while the induced upward FAC line the eastern edge of the $\Omega$. These FAC areas are connected by induced ionospheric currents $\boldsymbol{J}^{\text {ind }}$. Outside the $\Omega$ area the induced currents, both horizontal and field-aligned, are vanishingly small. The sum of the induced upward FAC is $\sim 80 \%$ of the downward FAC, so there is a small net downward induced current. In the input system there is considerably larger net upward current, for the sum of downward FAC is only about $50 \%$ of the upward FAC. As in the previous WTS case, also in the $\Omega$-band part of the induced FAC are situated in areas where the input FAC are not large. The induced FAC are not negligible, so 


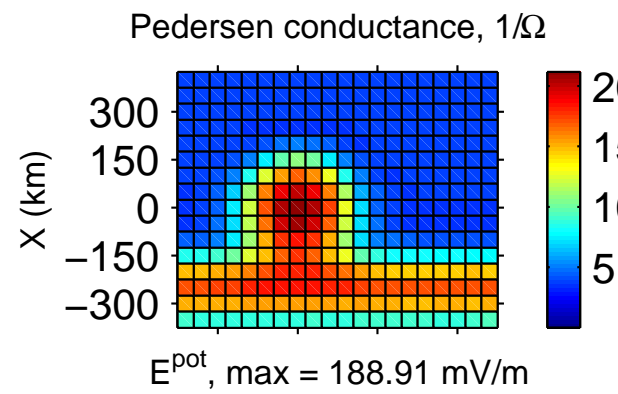

\section{Hall conductance, $1 / \Omega$}
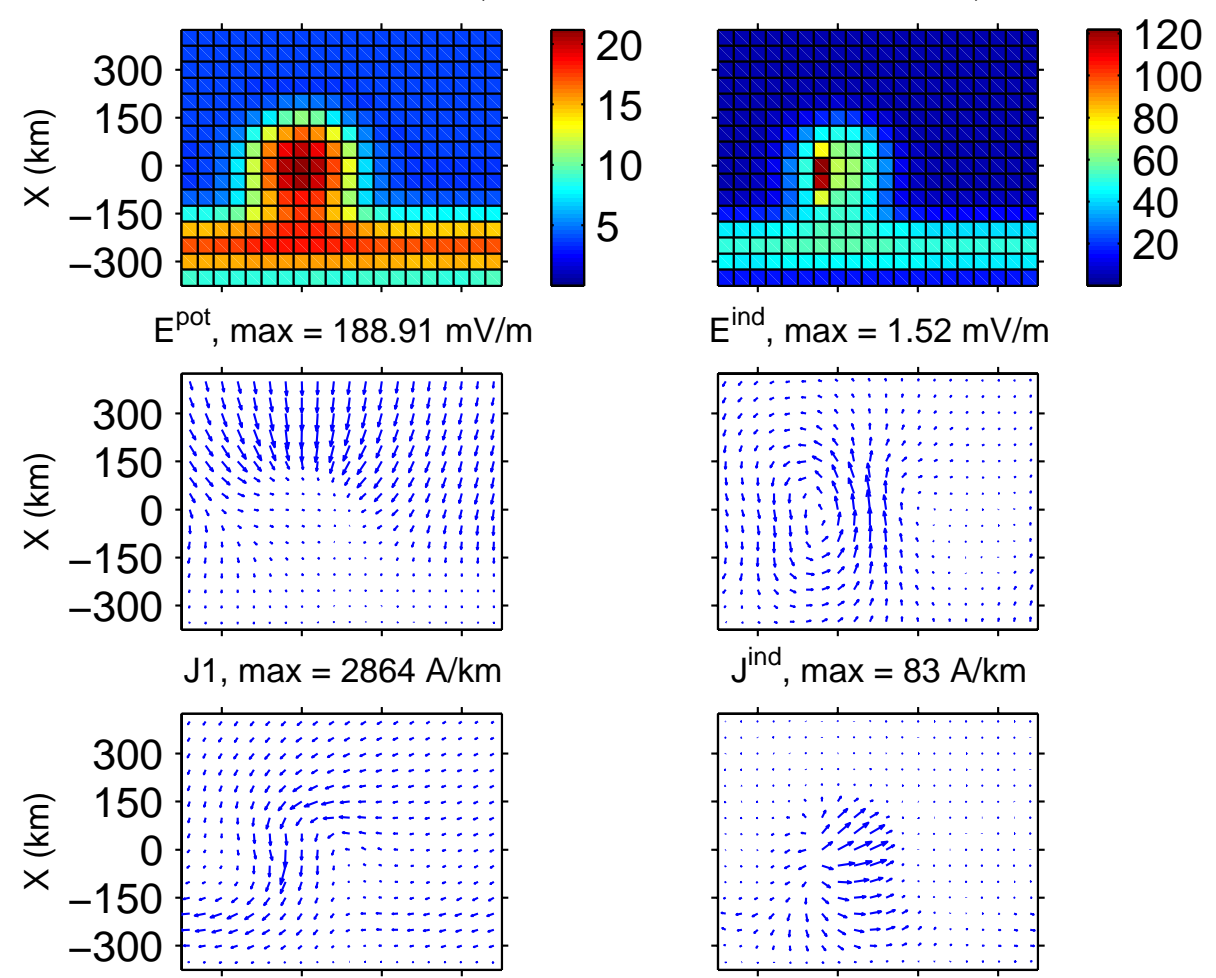

$\mathrm{FAC1}, \mathrm{A} / \mathrm{km}^{2}$

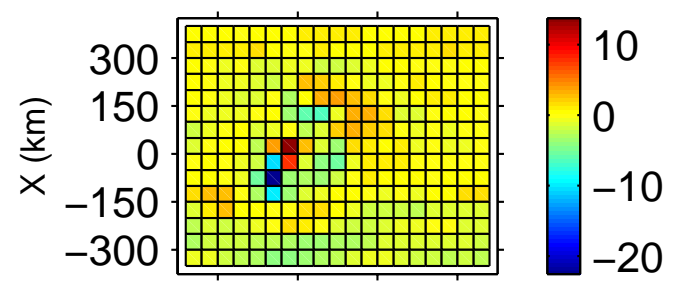

$$
F A C^{\text {ind }}, A / \mathrm{km}^{2}
$$
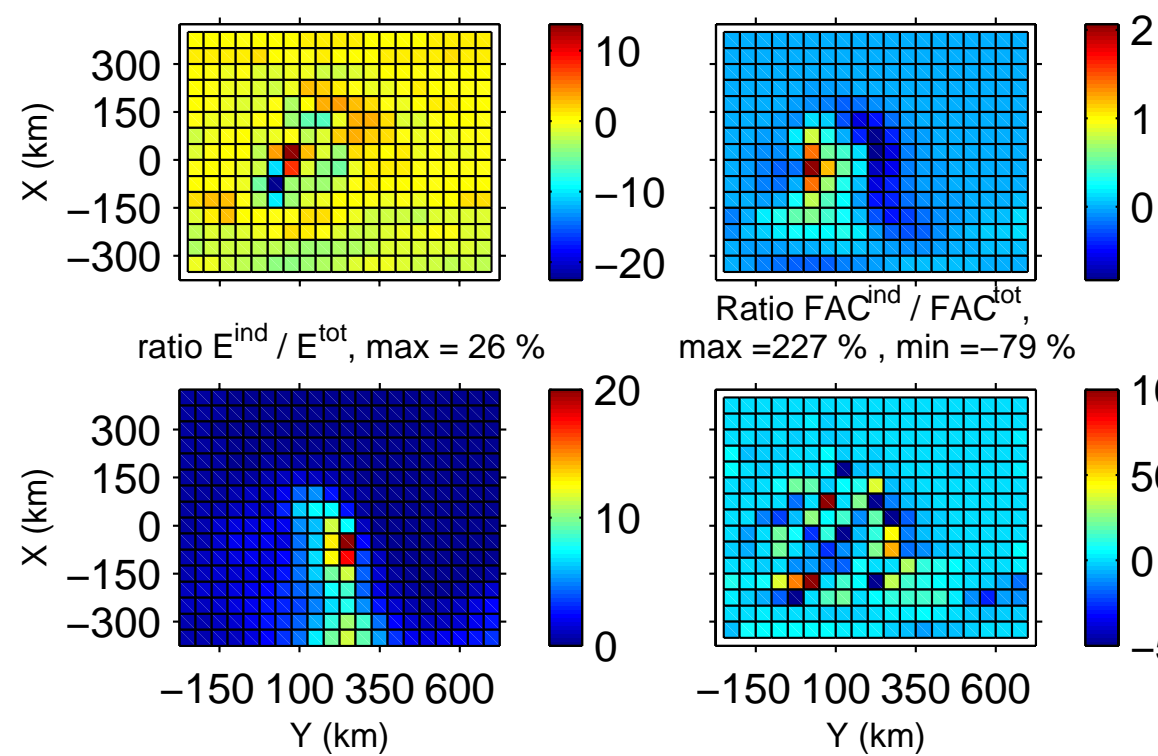

Ratio FAC ind $^{\text {FAC }}$ Ft $^{\text {tot }}$ $\max =227 \%, \min =-79 \%$
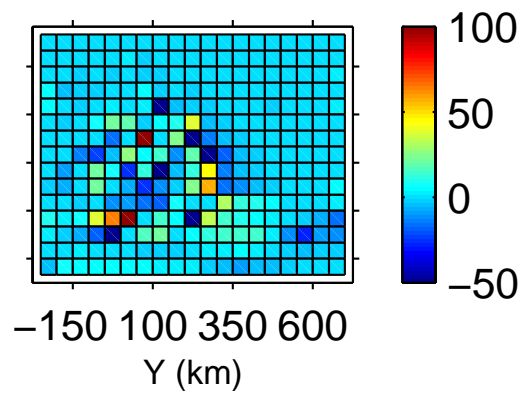

Fig. 13. Same as Fig. 5, but for the $\Omega$-band model.

this means that also in this case ionospheric induction may change the ionosphere-magnetosphere coupling.

Figure 15 shows a comparison of the induced electric field with the time derivative of the divergence-free input system. Again, there is a good resemblance between the vector fields, although not as close as in the WTS case. Typical length scale of $d(J 1 d f) / d t$ is about $150 \mathrm{~km}$ in this case, so Eq. (2) gives estimate $\left|\boldsymbol{E}^{\text {ind }}\right| \approx 11 \mathrm{mV} / \mathrm{m}$, which is again too high by a factor of 7. Comparison of Figs. 10, 15 and a similar plot for the electrojet case (figure not shown) reveals some common features. The basic shape of the induced electric field is estimated very well by calculating the time derivative 

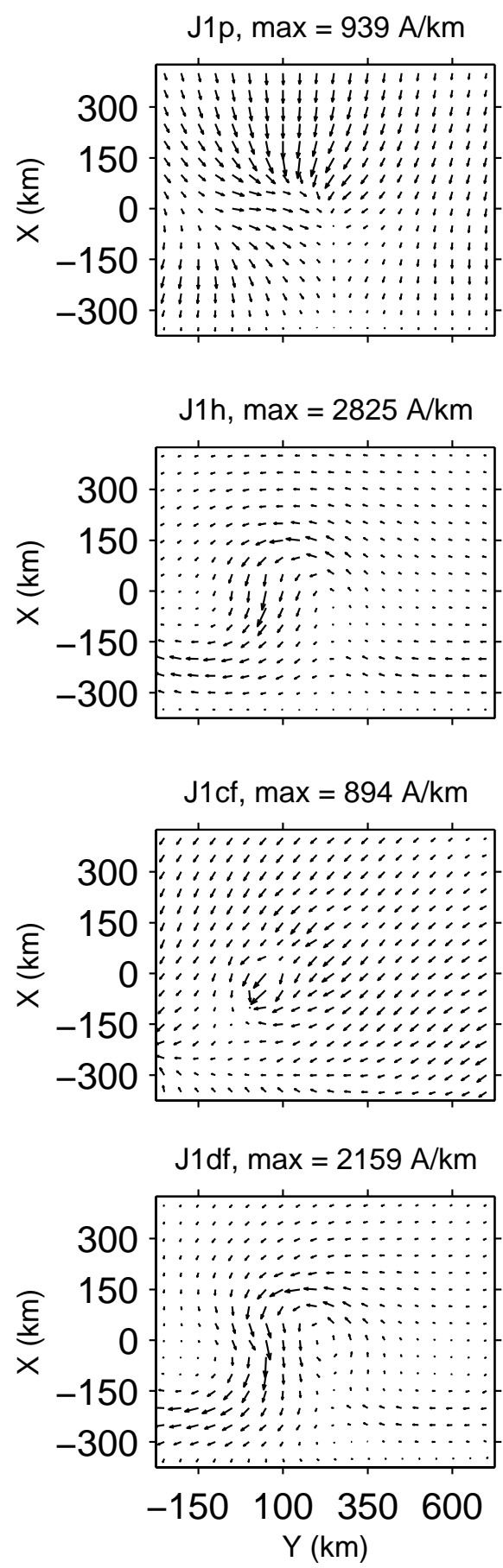
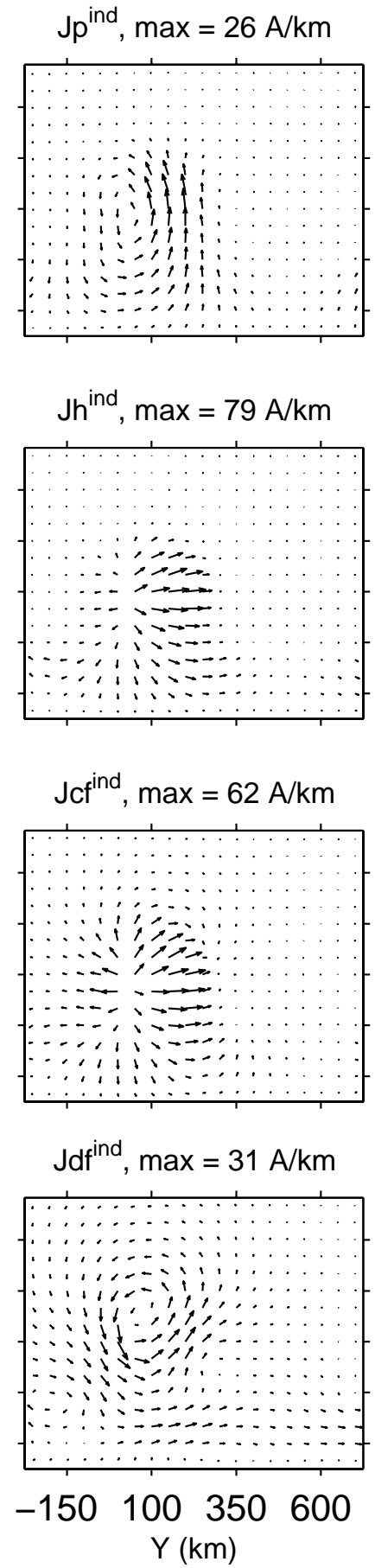

Fig. 14. Same as Fig. 6, but for the $\Omega$-band model.

of the divergence-free input currents. The exact calculation gives a somewhat smoother vector field, which also spreads out in a larger area than where $d(J 1 d f) / d t$ is concentrated. This kind of smoothing is expected, since the real induction process is non-local both in space and in time. Furthermore, estimates in Figs. 10 and 15 were calculated using only the divergence-free input currents, ignoring the induced divergence-free currents. Equation (2), with a somewhat arbitrary length scale estimate, may also be used to estimate the magnitude of the induced electric field. These estimates seem to be consistently too large by a factor of 5-10. 
The magnetic field associated with the induced currents of the $\Omega$-band is shown in Fig. 16. The induced B-field is in practise negligible in comparison with the original magnetic field. Only the induced horizontal $\boldsymbol{B}_{\perp}^{\text {ind }}$ above the ionosphere reaches values $\sim 6 \%$ of the input field. This reflects the fact that in the $\Omega$-band the induced horizontal currents are relatively small when compared to the currents in the input system, but the induced FAC have a somewhat larger impact. The relatively wide and smooth distribution of the input FAC also means that the associated B-field above the ionosphere is quite featureless. The induced B-field is more concentrated, so it is able to modify the magnetic signature of the $\Omega$-band above the ionosphere to some extent.

\section{Summary and conclusions}

We have calculated the induced electric fields and current that are present in some typical ionospheric systems. The calculation was performed using the method presented by Vanhamäki et al. (2006). In this calculation method the ionospheric potential electric field $\boldsymbol{E}^{\text {pot }}$ and height integrated conductances $\Sigma_{P}$ and $\Sigma_{H}$ are given as input. Output is the induced rotational electric field $\boldsymbol{E}^{\text {ind }}$ in the ionosphere. The main difference to previous methods, e.g. Yoshikawa and Itonaga (1996), Buchert (1998) and Lysak (2004), is that we do not have to specify the Alfvén waves incident from the magnetosphere, but can use more easily measurable ionospheric parameters as input. The calculation method of Vanhamäki et al. (2006) can be used with general, non-uniform and time-dependent conductance distributions, which enables us to use very realistic data-based ionospheric models, as is done in Sect. 4.

The simplified examples presented in Sect. 3 clarify the effect of ionospheric conductances and characteristic time scales on the induction process. In these examples, for the sake of clarity we assume uniform conductances, unlike in the realistic examples presented in Sect. 4. We find that the induced electric field depends on the ratios $\Sigma_{H} / T$ and $\Sigma_{P} / T$. Increasing the Hall conductance increases the induced electric field linearly and the induced FAC as $\Sigma_{H}^{2}$. The effect of a varying Pedersen conductance is smaller. With large $\Sigma_{P}$ the induced electric field is somewhat decreased in magnitude and becomes concentrated in a smaller area. The effect of varying oscillation time $T$ is a combination of the effects of varying $\Sigma_{P}$ and $\Sigma_{H}$.

We also considered three typical examples of ionospheric electrodynamic situations, namely an intensifying electrojet, a westward travelling surge and an $\Omega$-band. All these models are realistic and based on observational data. In the WTS and $\Omega$-band models the induced electric field is concentrated in a rather small area, where the temporal changes of the current system are largest. The induced field $\boldsymbol{E}^{\text {ind }}$ is quite significant in these "hot-spots", reaching values $20-50 \%$ of the potential field. Because the hot-spots are located in areas of
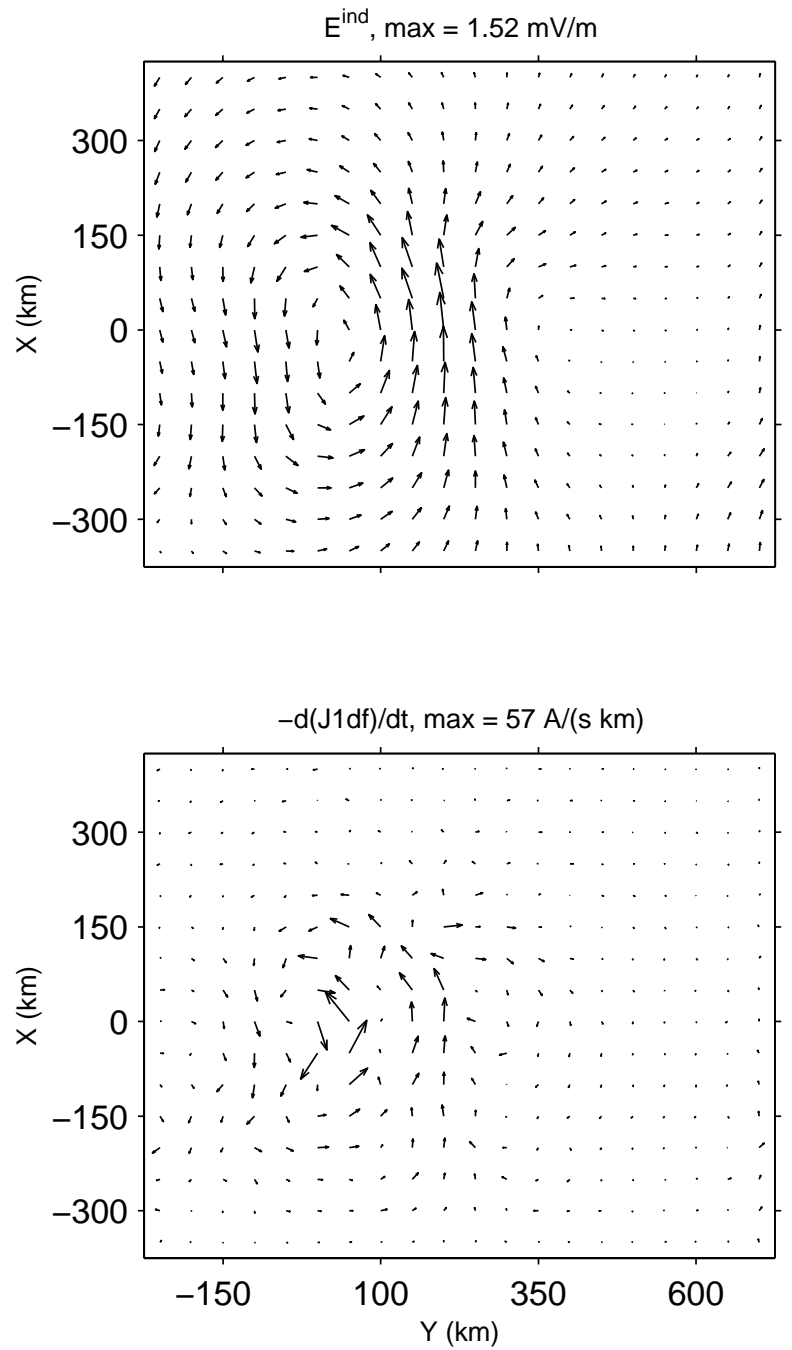

Fig. 15. Same as Fig. 10, but for the $\Omega$-band model.

enhanced conductances, even relatively small electric fields are associated with large ionospheric currents and FAC. In these two examples the induced currents form small localized loops, which modify the pattern of the otherwise present non-inductive currents. In the example of the non-uniform, intensifying electrojet the induced electric field has a magnitude of $\sim 10 \%$ of the potential E-field in large parts of the system. Also the induced FAC are spread in a large area and contribute about $20 \%$ of the total FAC in the electrojet. The induced currents are also associated with magnetic fields, that may have a significant contribution to the total B-field, especially above the ionospheric current sheet.

In the three realistic examples the general shape of the induced electric field was rather well approximated by calculating the negative time derivative of the divergence-free ionospheric currents, as in Eq. (2). The exact calculation method gave somewhat smoother vector fields that were also spread in a larger area, but the general structure was similar. With 


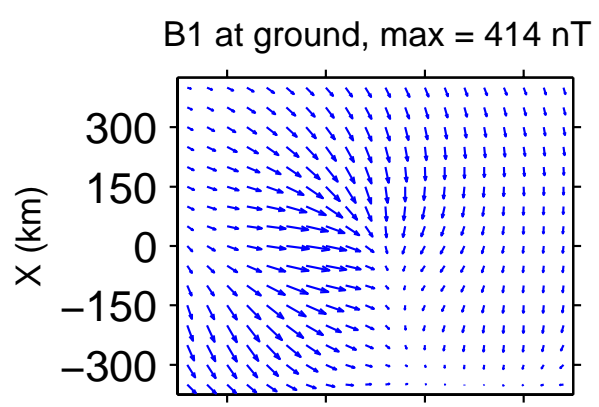

$\mathrm{B}^{\text {ind }}$ at ground, $\max =5.1 \mathrm{nT}$
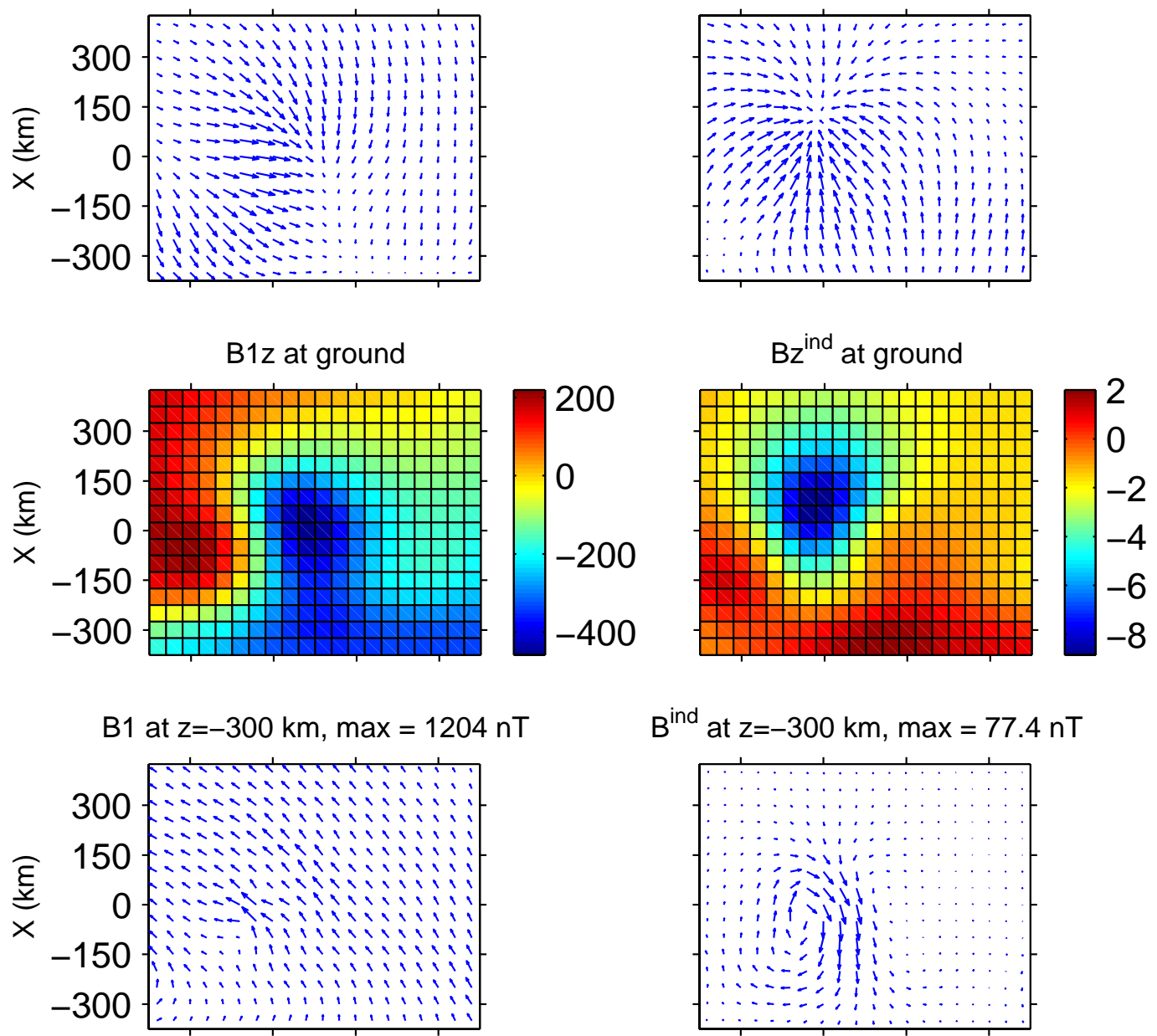

$\mathrm{B}^{\text {ind }}$ at $\mathrm{z}=-300 \mathrm{~km}, \max =77.4 \mathrm{nT}$
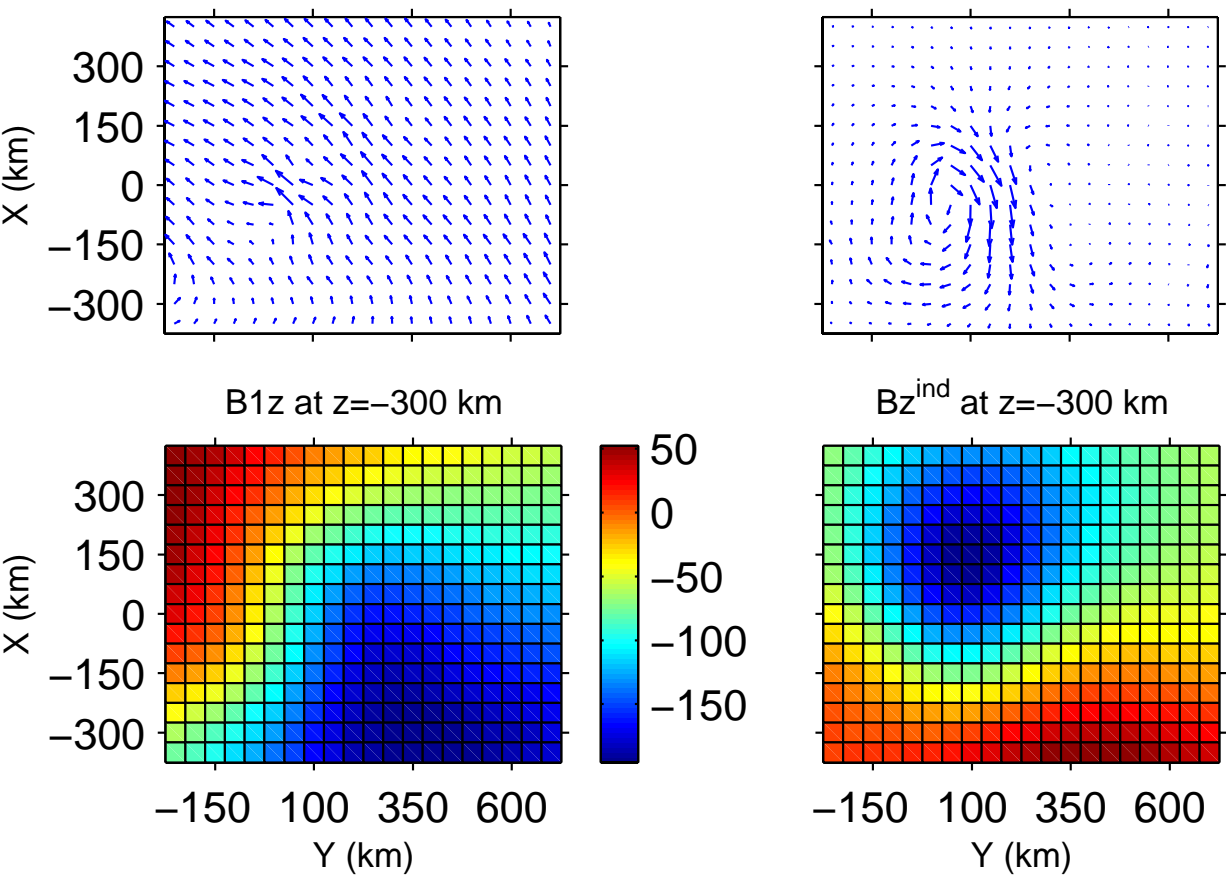

$\mathrm{Bz}^{\text {ind }}$ at $\mathrm{z}=-300 \mathrm{~km}$

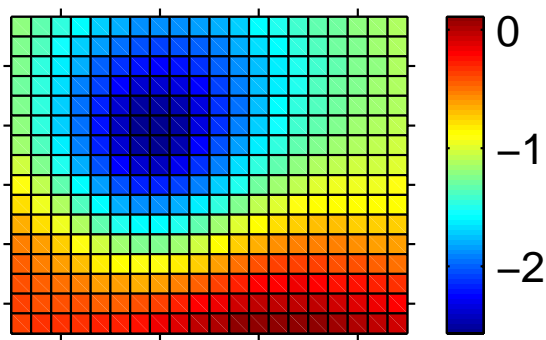

$\begin{array}{ccc}-150 & 100350600 \\ Y(k m)\end{array}$

Fig. 16. Same as Fig. 7, but for the $\Omega$-band model.

Eq. (2) the magnitude of the induced field was over-estimated by a factor of 5-10 in all three cases, although the rather arbitrary determination of a characteristic length scale may be part of the reason. Futhermore, the ionospheric divergencefree currents are almost the same as the ionospheric equivalent currents that may be estimated using ground based magnetometers (e.g. Untiedt and Baumjohann, 1993). This means that we may use Eq. (2) to estimate the significance of ionospheric induction in specific events just from magnetometer data. However, because Eq. (2) is rather approximate, events where inductive electric fields seem to be large should be studied with more care. 
Our results, although limited to three specific events, show that locally inductive phenomena have an important role in (terrestrial) ionospheric electrodynamics. Induced electric fields in the ionosphere change the structure of the pure potential field that is mapped from the magnetosphere along field lines, and also induced FAC alter the coupling between the ionosphere and magnetosphere. Inductive effects are largest in the most dynamical events, which are usually the most interesting ones.

The calculation method presented by Vanhamäki et al. (2006) that is used in this study assumes a 2-dimensional thin-sheet ionosphere. While this is a widely used and usually good enough approximation, the real 3-dimensional structure of the ionospheric currents may affect the induction process. A first step towards a 3-dimensional induction could be made by generalizing the calculation method presented by Vanhamäki et al. (2006) to incorporate two ionospheric current sheets at different altitudes. The upper and lower sheets would contain mainly Pedersen and Hall currents, respectively. Already this simplistic 2-layer model would contain new features, like mutual induction and current closure between the two sheets.

Another interesting future study would be to obtain observational data on the ionospheric induction process. One possibility would be to observe Alfvén waves at the magnetosphere both before and after they reflect from the ionosphere using the Cluster spacecraft. Simultaneous observations of the ionospheric reflection area by a network of groundbased radars and magnetometers would allow us to compare the measured properties of the reflected waves with theoretical models.

Acknowledgements. The work of H. Vanhamäki is supported by the Finnish Graduate School in Astronomy and Space Physics.

Topical Editor M. Pinnock thanks J. Vogt and another referee for their help in evaluating this paper.

\section{References}

Amm, O.: Direct determination of the local ionospheric Hall conductance distribution from two-dimensional electric and magnetic field data: Application of the method using models of typical ionospheric electrodynamic situations, J. Geophys. Res., 100, 21 473-21 488, 1995.

Amm, O.: Improved electrodynamic modeling of an omega band and analysis of its current system, J. Geophys. Res., 101, 26772683, 1996.

Amm, O.: Ionospheric elementary current systems in spherical coordinates and their application, J. Geomagnetism and Geoelectricity, 49, 947-955, 1997.

Buchert, S.: Magneto-optical Kerr effect for a dissipative plasma, J. Plasma Phys., 59, 39-55, 1998.
Buchert, S. and Budnik, F.: Field-aligned current distributions generated by a divergent Hall current, Geophys. Res. Lett., 24, 297 300, 1997.

Janhunen, P.: On the possibility of using an electromagnetic ionosphere in global MHD simulations, Ann. Geophys., 16, 397-402, 1998, http://www.ann-geophys.net/16/397/1998/.

Kamide, Y., Richmond, A., and Matsushita, S.: Estimation of ionospheric electric fields, ionospheric currents, and field-aligned currents from ground magnetic records, J. Geophys. Res., 86, 801-813, 1981.

Lysak, R.: Magnetosphere-ionosphere coupling by Alfvén waves at midlatitudes, J. Geophys. Res., 109, A07201, doi:10.1029/2004JA010454, 2004.

Lysak, R. and Song, Y.: A three-dimensional model of the propagation of Alfvén waves through the auroral ionosphere: First results, Adv. Space Res., 28, 813-822, 2001.

Lühr, H., Aylward, A., Buchert, S., Pajunpää, K., Holmboe, T., and Zalewski, S.: Westward moving dynamic substorm features observed with the IMAGE magnetometer network and other ground-based instruments, Ann. Geophys., 16, 425-440, 1998, http://www.ann-geophys.net/16/425/1998/.

Paschmann, G., Haaland, S., and Treumann, R. (Eds.): Auroral Plasma Physics, Space Sci. Rev., 103, 1-486, 2002.

Pulkkinen, A., Lindahl, S., Viljanen, A., and Pirjola, R.: Geomagnetic storm of 29-31 October 2003: Geomagnetically induced currents and their relation to problems in the Swedish highvoltage power transmission system, Space Weather, 3, S08C03, doi:10.1029/2004SW000123, 2005.

Richmond, A. and Kamide, Y.: Mapping electrodynamic features of the high-latitude ionosphere from localized observations: Technique, J. Geophys. Res., 93, 5741-5759, 1988.

Ruohoniemi, J. and Baker, K.: Large-scale imagining of highlatitude convection with Super Dual Auroral Radar Network HF radar observations, J. Geophys. Res., 103, 20 797-20 811, 1998.

Sciffer, M., Waters, C., and Menk, F.: Propagation of ULF waves through the ionosphere: Inductive effect for oblique magnetic fields, Ann. Geophys., 22, 1155-1169, 2004, http://www.ann-geophys.net/22/1155/2004/.

Untiedt, J. and Baumjohann, W.: Studies of polar current systems using the IMS Scandinavian magnetometer array, Space Sci Rev., 63, 245-390, 1993.

Vanhamäki, H., Viljanen, A., and Amm, O.: Induction Effects on Ionospheric Electric and Magnetic Fields, Ann. Geophys., 23, 1735-1746, 2005, http://www.ann-geophys.net/23/1735/2005/.

Vanhamäki, H., Amm, O., and Viljanen, A.: New Method for Solving Inductive Electric Fields in the Non-Uniformly Conducting Ionosphere, Ann. Geophys., 24, 2573-2582, 2006, http://www.ann-geophys.net/24/2573/2006/.

Yoshikawa, A. and Itonaga, M.: Reflection of shear Alfvén waves at the ionosphere and the divergent Hall current, Geophys. Res. Lett., 23, 101-104, 1996.

Yoshikawa, A. and Itonaga, M.: The nature of reflection and mode conversion of MHD-waves in the inductive ionosphere: Multistep mode conversion between divergent and rotational electric fields, J. Geophys. Res., 105, 10 565-10 584, 2000. 